Article

\title{
An Energy Efficient Routing Approach for Cloud-Assisted Green Industrial IoT Networks
}

\author{
Khadak Singh Bhandari (D) and GI Hwan Cho *(D) \\ Division of Computer Science and Engineering, Jeonbuk National University, Jeonju 54896, Korea; \\ khadak@jbnu.ac.kr \\ * Correspondence: ghcho@jbnu.ac.kr; Tel.: +82-10-341-25323
}

Received: 22 July 2020; Accepted: 1 September 2020; Published: 8 September 2020

\begin{abstract}
The green industrial Internet of things (IIoT) is emerging as a new paradigm, which envisions the concept of connecting different devices and reducing energy consumption. In multi-hop low power and lossy network, a resource-constrained node should aware of its energy consumption while routing the data packets. As part of IoT, the routing protocol for low power and lossy network (RPL) is considered to be a default routing standard. Recently, RPL has gained a significant maturity, but still, energy optimization is one of the main issues, because the default objective function (OF), which makes routing decision mainly based on a single parameter, such as link quality, and ignores the energy cost. Therefore, this paper aims to consider the concept of green IIoT concerning how a routing approach can achieve energy efficiency in resource-constrained IoT networks. For this, we propose a resource aware and reliable OF (RAROF), which constructs an optimum routing path by exploiting the information regarding the duty cycle, link quality, energy condition, and resource availability of a node. In addition, we propose node vulnerability index (NVI), a new routing metric that identifies the vulnerable nodes in terms of energy. To deal with the diverse data traffic of the IIoT network, we implement a multi-queuing based traffic differentiation approach that ensures the application requirements. The extensive simulation results show that the proposed RAROF can effectively extend the lifetime of the network, enhance the energy efficiency, and achieve higher reliability than that of other OFs. In this way, RAROF makes a routing decision with the purpose of extending network lifetime and minimizing energy depletion, paving the way towards green IIoT.
\end{abstract}

Keywords: energy efficiency; green IIoT; low power and lossy network; routing protocol; routing metrics; 6LoWPAN; duty cycle

\section{Introduction}

In recent years, we have seen considerable advances in terms of utilizing cloud platforms in the industrial Internet of things (IIoT) network architectures [1]. The emerging Internet of things (IoT) concept is expected to be a significant enabler for building the traditional industrial system more intelligent and efficient. Moreover, it also provides the opportunity to transform the traditional functioning of several existing industrial systems such as healthcare and manufacturing. To this end, IIoT plays an important role in improving and simplifying the way to monitor, manage, and control industrial processes through integrated sensors, actuators, data management, and analytics [2]. IIoT is an emerging heterogeneous networking paradigm equipped with various smart devices, sensors, and actuators designed for different industrial domains. Both the classic IoT and IIoT have many technologies in common such as machine-to-machine communication, data analytics, automation, and cloud platform, but they have different objectives, users, and operational processes. In general, IoT applications are more concerned with consumer usages, such as smart appliances, smart homes, and wearables, whereas IIoT is most commonly designed for industrial purposes such as healthcare 
systems, manufacturing, factory automation, supply chain management, and utilities. In short, the IIoT concept focuses mainly on improving efficiency, safety, and production [3]. Moreover, various industrial domains can greatly benefit from IIoT by saving time and cost through smart notification and alerts, early malfunction detection, predictive maintenance, improved safety, and performance monitoring. Furthermore, the cloud-assisted IIoT system enables the real-time performance analytics of the industrial system remotely, thereby optimizing the outcome.

It is emphasized that the realization of IoT systems in any industrial domain will exploit a large number of smart objects for enhancing the operation by enabling the accessibility and acquisition of a large amount of data. The data generated from a large number of connected smart objects is vital to the IIoT ecosystem as it can provide critical information for being industries more efficient, smarter, and sensible. To realize this, embedded devices, sensors, and actuators cover most of the critical areas and instruments, where these devices sense the environment, monitor the conditions, and collect the information to share between devices and with the control system. Considering the simplicity of these devices, the collected information can be treated by a central computing infrastructure, for instance, a cloud-based data center [4]. In the industrial networking settings, the low power and lossy networks (LLNs) are widely used for different applications and services such as condition monitoring, process automation, and environment sensing. Generally, the applications under condition monitoring cover the monitoring of mechanical, structural, and human conditions. Whereas, the industrial applications for environment monitoring include the problems of noise, water, air, temperature, humidity, etc. Likewise, the process automation applications cover the information regarding service delivery, including resource availability, current stock, demand, and supply process, etc. [5]. In this way, IIoT connects various objects to facilitates the complex tasks of monitoring, controlling, and automating industrial activities and provides efficient services with a new opportunity.

The industrial wireless network can be seen as a core physical infrastructure for industrial information systems (IIS), and collect data to support engineers and operators in the decision-making and crisis management. In IIoT networks, the generated data is large and important such as real-time monitoring, supervision, and control instructions, which need to be delivered timely and reliably. In such a circumstance, it indicates not only the presence of several traffic types within the network but also the different quality of service (QoS) requirements [6]. Moreover, IIoT networks may provide multiple applications and services, some of which are not only time-critical but also delay-tolerant. In this context, IIoT networks may face significant challenges to ensure the stringent requirements of industrial applications such as high reliability, energy efficiency, low communication cost, and low latencies. On the other hand, industrial wireless networks differ from traditional wireless networks in several ways. The devices of IIoT have limited resources in terms of energy, computation, memory, and wireless link. In the IIoT environment, with the help of other nodes (i.e., intermediate nodes or relay nodes), the source node transmits its data packets to the destination node (i.e., gateway or sink) due to the limited transmission range [7]. These characteristics bring new challenges to improve energy efficiency and to design a reliable routing protocol. Thus, to ensure the QoS requirements, the routing approach in the industrial network needs to determine reliable data transmission and also consider the node's constraints because routing decisions can influence overall network performance.

Currently, the industrial wireless network is still in the preliminary stages of network design, routing protocols, and deployment. However, IoT-based applications are having a significant impact on today's industries by leveraging cutting-edge technologies and standards. The WirelessHART, LoRa, and NB-IoT are some of the major wireless communication technologies used in the IIoT scope. WirelessHART and LoRa rely on an unlicensed frequency band, whereas NB-IoT uses a licensed spectrum. Because of the harsh environment and ultra-high reliability requirements of industrial wireless networks, these standards adopt the IEEE802.15.4 because they require a dedicated physical layer, and it supports many commercial off-the-shelf chips [5]. Furthermore, industrial network infrastructure can also be structured based on several enabling technologies such as cloud computing [8], big data [9], and Blockchain [10-12]. Since connectivity plays a crucial role in industrial 
network design to enable interconnection between heterogeneous systems, IIoT infrastructure relies on short-range wireless communications such as ZigBee, Wi-Fi, Bluetooth, and Radio-frequency identification [13].

In an industrial environment, connecting the physical working system with real-world network systems like the Internet or cloud is essential to enrich IIS. However, typical wireless sensor networks (WSNs) cannot interact with the Internet or cloud because some protocols of WSNs do not use the IP protocol [14]. Thanks to the contributions of the working group of IETF for IPv6 over low power wireless personal area networks (6LoWPAN) that made possible the integration of the constrained wireless network with an IP based network or the Internet [15]. It is standardized as the technique to implement IPv6 over IEEE 802.15.4 networks. 6LoWPAN is developed to enable an IoT device with IP communication functionality by adding the adaptation layer between data-link and network layer. As a result, the implementation of an industrial emergency monitoring system (IEMS) is possible by integrating IIS with the 6LoWPAN industrial wireless network. Furthermore, to provide the routing solution, IETF has developed a particular routing specification named IPv6 routing protocol for low power and lossy networks (RPL) to be used in resource-constrained networks such as 6LoWPAN [16]. In addition, RPL is intended to overcome different routing requirements of various application domains, namely urban application, industrial application, and home and building automation [17]. IoT devices run RPL to easily communicate with IP-based networks or the Internet through various links such as IEEE 802.15.4, power-line communication, and Bluetooth; therefore, RPL is often considered as routing protocol for IoT, and it can be applied in IEMS.

Since LLNs is an important infrastructure in the IIoT ecosystem, the main challenge is efficient resource utilization and the design of routing strategy. In multi-hop IIoT network infrastructure, the node performs a dual role as sender and forwarder; therefore, for each node, routing is the key functionality for efficient data delivery over a network. In addition, the routing protocol contributes to determining energy efficiency and communications reliability as well. Thus, for the IIoT network to be an energy-efficient, routing approach is an important part because routing protocol plays an important role in making efficient use of limited network resources. For most of the IIoT applications, the large number of sensor devices are typically deployed in harsh environmental situations, making it difficult to reach the device for battery recharge or replacement. Even though the life of the battery of any device is not endless, however, reducing the energy consumption for prolonging the lifetime of the network is an important aspect of routing optimization in the network. In resource-constrained networks, most of the energy consumption on a node occurs due to the transmission and reception, because the rest of the time, generally nodes are in sleeping or inactive state. While RPL is considered as a default routing protocol for IoT, it constructs the routing topology based on a different node and link metrics and constraints. The route selection in RPL is based on either a link quality metric or hop count, which results in an inefficient route selection in terms of energy efficiency because nodes are unaware of any energy resources. Furthermore, a single metric based topology construction approach in RPL makes the network imbalance and creates energy hotspots, particularly near to the root node. The best route selection is performed in such a manner to minimize a path cost based on specific metrics in the objective function (OF). Therefore, the routing decision in RPL is mainly made based on a single parameter, such as reliability, or delay. As a result, the selection of only one parameter in the routing decision inevitably suppress other. For example, if the only delay is considered, the nodes cannot experience high energy consumption, and vice versa. For this reason, metrics that represent multiple of these parameters need to consider in the OF to fulfill IIoT requirements, which is one of the goals of this paper.

Many existing studies have presented a different version of RPL energy optimization, but the duty cycling aspect has ignored mostly. According to the evaluation in [18], the default implemented version of RPL cannot encounter the QoS requirements of modern IoT concepts, such as resource efficiency, network lifetime, and network reliability. In order to enlarge network lifetime, efficient resource utilization of nodes is an important concern particularly for resource-constrained networks. 
Besides, for efficient data delivery in IoT applications such as emergency monitoring, the forwarding node should be aware of nodes' sleep and awake states. Based on this understanding, in this paper, we propose a resource aware and reliable OF (RAROF) based on RPL specification to enable the IIoT system more reliable and energy-efficient. For efficient data delivery, RAROF builds an optimum path by exploiting the information regarding the duty cycle, link quality, and energy condition of the node through which a node sends the data packets. Our contribution in this paper can be summarized as follows:

- This paper enhances the routing approach in RPL for the IIoT system with different data flows. For this, RAROF selects the reliable path focusing on the node resources. Unlike default path selection, we consider different routing metric that represents the node as well as link characteristics. Besides, these routing metrics are aimed to respond to the requirement of the IIoT applications.

- A new routing metric named node vulnerability index (NVI) is proposed, which includes the duty cycle information, link quality, energy profile of a node during the path selection process for network life improvement.

- We aim to put forward a simple conceptual cloud-assisted emergency monitoring system architecture for RPL based IIoT application. The composite architecture specifies the application of RPL by focusing on reforming industrial operations through cutting-edge technologies.

- We propose a simple multi-queueing approach to deal with the diverse data traffic generated from the IoT application. In this perspective, the queuing model is responsible for employing the prioritization of each packet according to the traffic sensitivity.

- We analyze the effectiveness of our proposed approach in different aspects by conducting extensive simulation tests through the Cooja simulator.

The rest of the paper is organized as follows: Section 2 presents the design issue and routing challenges in IIoT networks. The recent related research work is presented in Section 3. The preliminaries of the work are presented in Section 4. Section 5 describes the proposed work in detail, and also provides the cloud-assisted IEMS architecture. Section 6 presents the experimental setup, simulation study, performance evaluation of the proposed RAROF with the comparisons, and discusses the key points of the proposed work. Finally, Section 7 concludes the paper.

\section{Design Issues and Routing Challenges in Green Industrial IoT Networks}

Industrial wireless networks are deployed with self-organizing, flexibility, and inherent smart processing ability. In order to make the industrial network green, the underlying communication technologies and routing strategy need to optimize that can satisfy the energy hunger of resource-constrained smart devices. In this paper, the green IoT concept is considered in a viewpoint of how the routing approach can achieve energy efficiency in the resource-constrained IoT system. While state-of-the-art technologies being considered, the industrial wireless network still presents different challenges, for instance, unstable communication links, limited resources (low bandwidth, storage, energy, etc.), and varied traffic pattern. These unique characteristics pose a significant challenge to the design of reliable and efficient routing solutions. Based on the purpose and requirements of the application, routing protocols in IIoT networks may face the following challenges:

\subsection{Wireless Links and Communication Patterns}

Harsh environment in industries are prone to high RF interference due to presence of several equipment, human, and obstacles. This adverse condition may result in wireless link instability and path disruption, which severely impact on data transmission of IIS. Furthermore, this situation leads to increased packet error rate and delay. Based on the application characteristic, the range of link delay may vary from a few seconds to minutes. On the other hand, different wireless communication technologies are used in the IIoT system; for energy efficiency, routing overhead should be reduced. 
The underlying routing protocol is the key player for path selection should address this instability and maintain reliable communication path.

The heterogeneous nature of industrial networks and applications indicates the presence of different communication patterns, such as point-to-point, point-to-multipoint, and multipoint-to-point. The presence of different communication patterns imposes challenges to data collection and distribution in an industrial environment [19]. Reliable data delivery is an important aspect in IEMS because the data can be transferred for different purposes, such as real-time monitoring, remote control, and emergency response.

\subsection{Resource Constraints and Interoperability}

IoT devices have limited resources in terms of energy supply, storage capacity, processing power, and transmission capacity due to their physical design conditions. Because of the hardware constraints, the routing approach should be implemented with minimal code and require a low routing state to be suitable for the device capacity. As a result, the routing design and implementation of IIoT concept are subject to some restrictions. In this regard, 6LoWPAN technology enables nodes to reduce power consumption and improve robustness due to its low complexity. Overall, these limitations are reflected in the protocol performance, so a lightweight routing protocol with low computational and energy requirements are needed. Moreover, some use cases in IIoT require users to deploy a large number of sensing and monitoring devices to be effective. Node placements range from hundreds to thousands, and the minimality should be considered for maximum benefit and utilization of resources. Therefore, seamless interaction with external networks (i.e., cloud or Internet) infrastructure is essential for making the IEMS more intelligent and pragmatic. Different techniques, such as in $[20,21]$, can be adopted to overcome the energy challenges of routing algorithms.

\subsection{Data Heterogeneity and Flow}

Different applications may need to simultaneously transmit different types of data (critical, non-critical, real-time, etc.) over the same link and to the same destination. In some cases, the traditional wireless networks are assumed to be homogeneous in terms of the data generated by the devices, but IIoT networks are heterogeneous. In IEMS applications, it is normal to have multiple devices installed in different places for specific purposes. Hence, it generates different data traffic depending on the use cases, such as heat monitoring, water and gas leakage, emergency alarm, fault detection, etc. This requires that the underlying communication mechanism reliably handles data delivery with specific requirements in terms of service availability. An efficient channel assignment algorithm and an interference-aware routing scheme could be a solution to some extent for the heterogeneous nature of IoT networks [22].

\section{Related Works}

\subsection{QoS Provision in Resource-constrained Networks}

The extensive use of the IoT concept has contributed to the presence of RPL, which enables smart devices to interact or communicate in the surrounding ambient at anytime and anywhere. Recently, many studies have been conducted on the various aspect of RPL optimization, such as congestion control [23], data aggregation [24], security and mobility [25,26], and OF design [27]. While RPL significantly satisfies the requirements of LLN for IoT based applications, to be fit in the IIoT concept, some aspects are crucial, such as energy efficiency and reliable routing. Due to the unique characteristic of wireless networks, it is difficult to define QoS parameters, and also poses additional challenges for QoS support. Therefore, to overcome these challenges, the authors in [28,29] provide different solutions for various application domains. There are two common approaches for QoS provisioning one is layered, and the other is cross-layered. In the individual layer, QoS provisioning depends upon layer capability. The layered approach provides the QoS with protocols that operate merely in each 
layer of the communication protocol stack, whereas the cross-layered approach achieves the QoS provisioning through the interaction of multiple layers concurrently. However, there is no variation between both approaches in terms of QoS support. The authors in [30] have conformed the widespread use of QoS provisioning with service differentiation compared to deterministic based QoS provisioning approaches. Therefore, most protocols follow the classification of packets based on traffic type, and packets from different classes are processed according to their requirements by tuning the MAC layer parameters. The traditional methods based on the resource reservation, route recovery during topological changes, and end-to-end path discovery are not suitable in LLNs for several reasons.

Based on this understanding, in the IIoT networks, it is essential to facilitate prioritization among different traffic classes when designing routing algorithms. This characteristic could be considered through different mechanisms. For instance, a priority queue system could be applied to differentiating the traffic with different QoS requirements. On the contrary, the mechanism implemented for QoS provisioning can exploit the multipath routing system. The authors in [31] presented a comprehensive survey on multipath routing protocols for QoS assurance. The study primarily focused on real-time multipath routing techniques for wireless multimedia sensor networks. In terms of QoS, a recent study shows that unlike single-path routing protocols, multipath routing protocols are suitable for WSNs. In fact, multipath routing protocols reduce the possibility of packet loss while utilizing redundant paths to the root [32]. Therefore, this mechanism can be used for a reliable-sensitive network where reliable information forwarding is essential, whereas real-time applications where delay-sensitivity is considered as basic QoS support this system may violate the specific application requirement. Multipath forwarding optimization has been proposed in [33] to minimize the average delay towards the root node based on the composite metric. The proposed work points out that default single-metric-based OFs are vulnerable in situations where congestion occurs due to a sudden increase in traffic volume, resulting in packet loss and delay. In this context, multipath forwarding techniques are desirable in LLN environments to achieve the desired QoS. However, some issues should be considered carefully while designing a routing protocol. Some of the issues about multipath approaches are high complexity, fragmentation, and overhead. Moreover, the high overheads incurred by multipath techniques in terms of communication and computation is unsuitable for devices with limited resources as well as for large-scale networks like the IIoT.

\subsection{Emergeing Technologies for Resource-Constrained Networks}

With rapid technological development, the new green IoT Concept aims to realize a sustainable smart world by reducing the energy consumption of the IoT ecosystem. The authors in [34], review the green information and communication technology (ICT) principles, and summarize the contemporary developments regarding future and current sensor-cloud. Moreover, future research directions and open issues related to green IoT has been presented. Turn off facilities that are not needed is the one widely used green ICT principles. Sleep schedule is the key technique for saving energy consumption by allowing nodes to wake up and sleep dynamically. With this understanding, we can conclude that green IoT focuses mainly on energy efficiency in the IoT ecosystem. Recently, in [2], many technological solutions have been proposed for implementing green IoT. Likewise, the authors in [35] presents the comparative analysis of different green IoT approaches. Besides, the energy efficiency models have been categorized based on the used technologies. Regarding the energy-efficient deployment of IoT devices, the authors in [36] have presented a framework for implementing the green IoT.

On the other hand, the sensor-cloud concept is attracting great interest from the research community, paving the way towards green IoT. It is a new concept, aimed to implement as an interface between the physical and cyber worlds with the data collection and processing capabilities of wireless networks. The basic model of the cloud-sensor is to collect various sensing data from the surrounding ambient and transmit to the cloud for further processing and storage. The authors in [37] explained the motivation behind using the cloud sensor. The concept is fundamentally a cloud platform for the collection, storage, 
and analysis of a large amount of sensed data in a seamless manner. Cloud integration has many promising advantages, which can contribute to the benefits for users and industrial wireless networks.

\subsection{RPL Optimization for Resource-Constrained Networks}

In the RPL scope, route selection is primarily based on a single metric, which may lead to an unbalanced network load. The overloaded node may consume more energy, thus draining their battery quickly than others, which eventually causes a network partition; this results in a significant impact on overall network performance and QoS requirements. Hence, an energy-oriented routing approach for QoS support is one of the key challenges in the resource-constrained network like IIoT. In this regard, some energy optimization approaches for RPL implementation have been presented by the research community in the recent years. Most of the work focuses on designing the OF that includes new energy related routing metrics.

The authors in [38] proposed an energy efficient and reliable composite metric for RPL organized networks. The proposed composite metric considers both energy and reliability parameters to select the optimum path. It takes into account the reliability through link metric, i.e., expected transmission count (ETX), and balances the network energy consumption through considering the nodes' energy availability. To make the energy-efficient and reliable RPL, the proposed metric named lifetime and latency agreeable metric $\left(\mathrm{L}^{2} \mathrm{AM}\right)$ allows the nodes to select the best parent by avoiding the congested one by estimating the traffic. However, it ignores the duty cycle status of a node during the parent selection process. In addition, the congestion alleviation process can cause frequent parent change in the network, making the network unstable, which is not appropriate for emergency monitoring IIoT network.

The authors in [39] proposed a virtual version of the RPL protocol, referred to as optimized multi-class RPL (OMC-RPL), based on a software-defined networking approach and virtualization. OMC-RPL focused on designing the enhanced OF with a composite routing metric. For this, it combines four weighted metrics to support multi-class traffic using software-defined networking (SDN)techniques. The weights of the metric are determined using the particle swarm optimization algorithm. Out of four metrics, the energy metric is considered as a secondary metric (optional) and therefore used whenever required. Even though the OMC-RPL considers different metrics, including the energy parameter, this virtual version of RPL may not fulfill the IIoT application requirements because it limits the energy profile of the nodes.

Recently, a new mobility-aware and energy-efficient parent selection method for LLNs has been proposed in [40]. The parent selection scheme select the optimum parent based on different routing metrics, such as ETX, expected lifetime, received signal strength indicator (RSSI), and distance of the mobile node and the parent node. Additionally, the authors proposed an optimized version of the Trickle timer called dynamic Trickle. This parent selection method mainly focuses on the issue of switching from one parent to another due to mobility. To prolong the network lifetime and minimize the energy use in smart grid networks, the authors in [41] proposed the new routing mechanism. For this, an efficient routing metric named energy and congestion-aware routing metric (ECRM) has been designed. ECRM considers the nodes' queue and residual energy information to select the next-hop node towards the root. To improve overall network performance, the node simply selects a parent node with a less queue occupancy and high remaining energy. However, finding such a node is not always be feasible. Different metric composition techniques have been presented in the literature to enhance the OF implementations in RPL and optimize the network resources. In this regard, the authors in [42] presented a new objective function named context-oriented OF (COOF) to utilize the network resources efficiently. COOF selects the best route based on the fuzzy logic technique using contextual information in which the node's queue, energy, and link status are considered as resource context. Another enhancement of RPL to satisfy the challenges imposed by the Internet of multimedia things has been proposed in [43]. The authors proposed a new version of the RPL routing protocol named green-RPL to enable green communication for multimedia connected networks. Such 
energy-efficient routing solution reduces the network carbon footprint emissions by ensuring the QoS requirements of the multimedia application. In green-RPL, a node selects the preferred parent based on the set of network metrics, including battery consumption and energy source types. In terms of packet delivery, the green-RPL is better as compared to the default RPL OFs; on the other hand, green-RPL is less energy efficient than ETX.

In [44], the authors have proposed a new energy consumption(EC) aware RPL-compliant OF using fuzzy logic named OF-EC. It combines multiple metrics such as ETX, hop count, and energy consumption to deal with the limitations of the single metric based RPL implementation. Although OF-EC combines node and link metric using membership function to select a parent node, it has neglected the resource utilization of a node, whilst it is one of the main concerns for resource-constrained networks. Similarly, the authors in [45] have introduced a new OF for efficient routing (ER) called OF-ER. To select the best parent, OF-ER uses the composite efficient routing metric. This metric takes into account multiple metrics, namely, ETX, queue utilization, delay, lifetime of node, and number of bottleneck nodes. However, it simply combines these metrics regardless of the isotonicity and monotonicity characteristics of the routing metrics.

Although energy metrics have been widely used in RPL optimization, there have been limited studies on consideration of duty cycling for energy profiling during routing metric design. Moreover, most of the above RPL extensions adopt the residual energy of a node, either alone or in combination with other nodes or link metrics, so as to choose the best path. Besides, RPL enhancements have been made either for specific purposes, such as congestion control, mobility or designed to support various protocol functioning using techniques such as fuzzy logic, SDN, etc. Reliable data communication in order to ensure the ideal emergency monitoring system in different industrial operations is essential, in which the IIoT network may generate various types of data traffic such as time-critical and delay-tolerant. This backdrop motivates the authors to design the new composite routing metrics and OF to optimize RPL for the IEMS use case of IIoT. In order to extend the network life through reliable data delivery for the resource-constrained network, not only the resource availability is important but also the nodes' sleep and awake status is vital. Moreover, heavy computational systems like fuzzy logic, particle swarm optimization may degrade the performance of a resource-constrained network. Therefore, to distinguish the efficiency of the RAROF, we have considered fuzzy logic-based OF-EC and resource-aware OF-ER as benchmarks for the comparison.

\section{Preliminaries}

In this section, we introduce an overview of the RPL routing protocol and the IEEE 802.15.4 standard in brief.

\subsection{IEEE 802.15.4 Based Low-Power Wireless Networks}

The IEEE 802.15.4 standard is considered to be fit for constrained networks in the IIoT domain because it allows low-rate low-cost communications [46]. It defines the media access control (MAC) and PHY layers for low-rate wireless personal area networks. This standard is the basis for many other technologies, such as WirelessHART and ZigBee. Furthermore, it is also used with RPL and 6LoWPAN for energy efficient operation. IEEE 802.15.4 based network supports two types of node full function devices and reduced function devices and can form a star, point-to-point, and cluster-tree topology. Principally, IEEE 802.15.4 defines two modes of operation, i.e., beacon-enabled and non-beacon, in which both operations make use of the carrier sense multiple access/collision avoidance (CSMA/CA) mechanism for channel access. In non-beacon-enabled mode, each node access the channel using a typical unslotted CSMA/CA technique, whereas beacon-enabled mode employs the superframe structure for channel access with slotted CSMA/CA mechanism. In our proposition, we take into account the beacon-enabled mode of operation because it enables an active and inactive section of the beacon interval for energy-efficient operation. Hence, in the following, we only describe slotted CSMA/CA and the superframe structure. 
Even though IEEE 802.15.4 protocol supports three different network topologies, beacon-enabled mode functions only in star or cluster-tree topology. The router or coordinator periodically transmits beacon frames to end devices to ensure node synchronization. This synchronization allows the device to sleep between coordinated transmissions to avoid idle listening, which results in increasing the life of the network. Since the beacon frames are broadcasted periodically, each beacon interval is divided into an active period, called a superframe, and an inactive period in which the node is in a low power state. Based on the duration of these two sections, the duty cycle of a node is determined. Figure 1 show the superframe structure of IEEE 802.15.4.

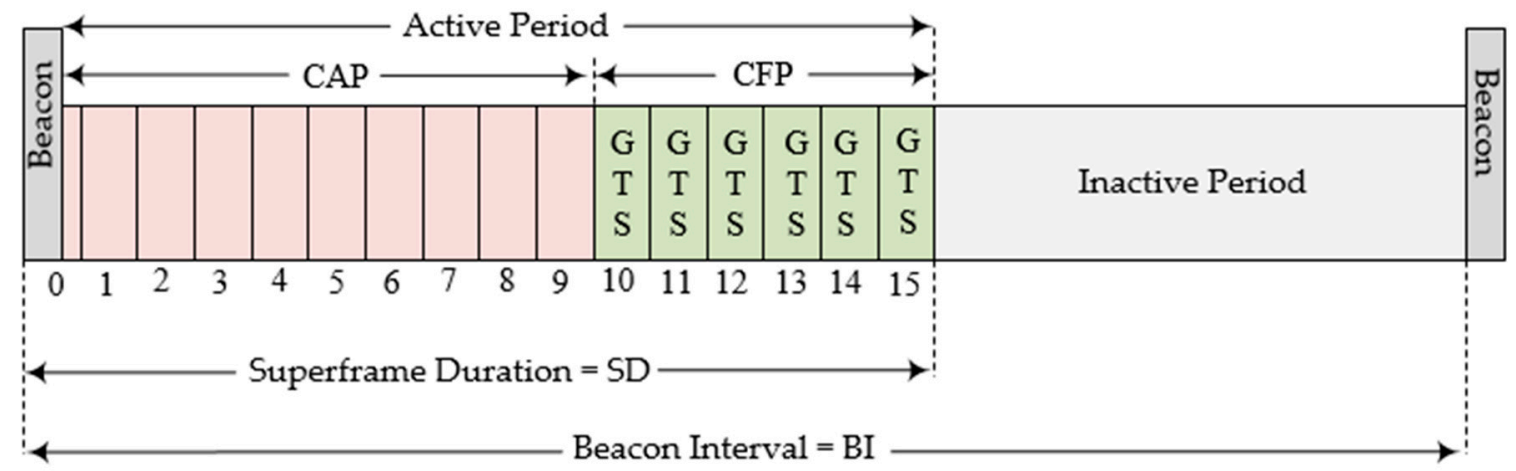

Figure 1. Superframe structure of IEEE 802.15.4.

The superframe structure is bounded by beacons and divided into equal 16 slots. The coordinator or router defines the format of the superframe based on the two fundamental parameters, which are beacon interval (BI) and superframe duration (SD). The coordinator transmits the next network beacons at the end of the $\mathrm{BI}$, so $\mathrm{BI}$ is the time duration between two consecutive beacons. The $\mathrm{BI}$ is divided into two portions active and inactive period. The active portion of the BI duration is known as the SD in which frames can be transmitted. Further, the SD is composed of the fixed sized slots of a contention access period (CAP) and a contention free period (CFP). During CAP, any node wishing to communicate shall compete to access the channel using a slotted CSMA/CA mechanism. On the other hand, during CFP, no transmission use CSMA/CA to access the channel. IEEE 802.15.4 standard allows nodes to use their reserved slots by providing guaranteed time slots (GTS) for QoS demanding applications. In our proposition, to provide the emergency monitoring service guarantee we use GTS for transmitting real-time traffic. To save the energy nodes can sleep during inactive period and the router define this period by selecting $\mathrm{BI}$ must be greater than $\mathrm{SD}(B I>S D)$. The length of $\mathrm{BI}$ and $\mathrm{SD}$ is determined by the beacon order $(\mathrm{BO})$ and superframe order $(\mathrm{SO})$ as follows.

$$
\begin{aligned}
& B I=\text { aBaseSuperframeDuration } \times 2^{B O} 0 \leq B O \leq 14 \\
& S D=\text { aBaseSuperframeDuration } \times 2^{S O} 0 \leq S O \leq 14
\end{aligned}
$$

where aBaseSuperframeDuration is the number of symbols defined by the standard based on the data rate and frequency. Therefore, it denotes the minimum superframe duration corresponding to $S O=0$, which is $15.36 \mathrm{~ms}$ while assuming $2.4 \mathrm{GHz}$ frequency band and $250 \mathrm{kbps}$ rate. Therefore, in our proposal, we consider this for defining the superframe duration. When non beacon-enabled mode is selected both the $B O$ and $S O$ is set to 15 and the router will not send any beacons and guaranteed service is not permitted.

\subsection{RPL Routing Specification}

RPL is a distance vector routing protocol that makes use of IPv6 based addressing technique and operates on top of the IEEE 802.15.4 and supports various types of link-layer technologies such as ZigBee and Bluetooth. It is capable of constructing a robust tree-like topology that connects hundreds of resource-constrained devices in the network, typically known as a directed acyclic graph (DAG). 
Each node in a graph connects in a way where there exist no cycles. The DAG is destined to one or more root and generally known as destination oriented DAG (DODAG). The DODAG root constructs and maintain the network through different control messages. For this, RPL specifies three main Internet control message protocol version 6 (ICMPv6) messages and exchange the network-related information. These messages are DODAG information object (DIO), DODAG information solicitation (DIS), and designation advertisement object (DAO). The DODAG root node initiates the topology formation process by broadcasting the network information through the DIO message. Once the DIO message is received by the neighboring nodes, they decide whether to join the network or not, based on the OF and policy. Afterward, the node adds the address of the DIO sender in its candidate parent table and calculates the rank, abstract distance between a node and the root node, based on the defined metric and $\mathrm{OF}$, which must monotonically increase towards the leaf node. Each node selects the preferred parent (next hop towards the DODAG root) from the candidate parent set that has the best rank. In this way, the upward path from leaf nodes to the root node can be established. In RPL scope, it is not mandatory that OF should use a particular routing metric, consequently, the OF can be defined according to the application requirements. Technically, we can use OF for two purposes, the rank calculation and optimum path selection based on routing metrics and constraints. As described in [16], different node and link metrics can be used during topology construction and route selection. Therefore, it is possible to use multiple routing metrics to fulfill the application goal, which is one of the objectives of this paper.

\subsection{Integration Between RPL and IEEE 802.15.4}

Our proposal attempts the coupling of the RPL routing protocol and the beacon-enabled IEEE 802.15.4 to support different QoS requirements of the emergency monitoring IIoT application. This integration enables efficient data delivery while reducing the energy consumption. These standards operate in two different layers therefore the network structure maintained by RPL is different with topology required in IEEE 802.15.4. In RPL structure, one node may associate with multiple parent nodes, while in IEEE 802.15.4 multi-hop structure, a node can associate with only one parent node. As a result, this structure may suffer from the beacons collisions while networking synchronization. Therefore, the superframe needs to schedule in a way that collision-free slots can be assigned and handling the overlapping superframes. Therefore, in our proposition, we implement the simple distributed beacon scheduling algorithm presented in [47]. Basically, the algorithm assigns the superframe slots randomly as well as on a greedy basis.

\section{Proposed Energy Efficient and Reliable Routing Approach}

In this section, we first present a general idea of the network model and assumptions related to the energy-efficient routing approach. Following this, we describe the routing metric and OF to support the traffic differentiation for the IIoT network, employing the RPL routing strategy.

\subsection{Network Model and Assumptions}

We consider an IIoT based emergency monitoring network scenario in RPL that consists of a number of LLN devices communicating in a multi-hop manner and form the directed acyclic graph. The devices in the network are deployed as $V=N \cup R$, where $N=\left\{n_{1}, n_{2}, n_{3}, \ldots, n_{n}\right\}$ denotes the set of nodes that can generate and transmit data packets, and $R$ denotes the set of all DODAG roots that can collect the data packets generated by the network. The whole network is modeled as a directed acyclic graph $G=(V, E)$, where $V=\left\{v_{1}, v_{2}, v_{3}, \ldots, v_{m}\right\}$ is the set of all the devices in the network, and $E$ is the set of all communication links among these devices. A connection link $e$ exists between nodes $\left(e\left(v_{i}, v_{j}\right) \in V\right)$ if $v_{i}$ and $v_{j}$ are in the transmission range of each other. The proposed monitoring network is heterogeneous in which resource-rich routers manage each of the DAG. Each node in the DAG connects in a way that the tree-shaped topology is formed. If the node is configured as a router, the node sends updated DIO messages to its neighbors to join the network. Otherwise, if the node is configured as a normal node, it simply joins the network and operates as a leaf (child node) 
in a tree to send their sensing information to the router (parent node). In a DAG structure, unlike a typical tree structure, a node can have multiple parent nodes. The node selects one as a preferred parent from the parent set. We assume that $N$ devices are distributed randomly in the monitoring network region controlled by the DODAG roots $R$. The transmission power range of every node is equal, and each node in the network is identified by a unique identifier, for example, an IPv6 address. Thus, a network is fully connected, and data can be sent from one node to another in a multi-hop manner. We also assume that the LLN devices are stationary for their lifetime. In regards to the neighbor discovery in the network, we consider the IPv6 neighbor discovery protocol. Furthermore, at any time, each LLN device can compute its energy level and has precise information about their local state (state information about all outgoing links). For this, we use the energy estimation module of Contiki OS. The entire tree-shaped network is divided into two DODAGs, each having a root, which is responsible for controlling the routing. Each DODAG root performs data aggregation and forwards it to the local cloud for further action. The routing path $R p$ from source node $v_{i}$ to destination node $v_{j}$ is represented as $R p\left(v_{i} \rightarrow v_{j}\right)=\left\langle v_{i}, v_{i+1}, v_{i+2}, v_{i+3}, \ldots, v_{j}\right\rangle$, in which $R p\left(v_{i} \rightarrow v_{j}\right) \in \mathbb{R}$. Here, $\mathbb{R}$ is a finite set of all the potential routing paths in the network. In a routing path, $v_{x}$ are nodes, such that $i \leq x \leq j$. If all nodes from $v_{i}$ to $v_{j}$ are distinct, then the routing path $R p$ is simple. Otherwise, if $v_{i}=v_{j}$, then it is the form of a loop in a routing path $R p$. For more detail, please refer to [16].

\subsection{Primitive Requirements for Routing Metric}

The use of the RAROF is to optimize the RPL performance for emergency monitoring IIoT network, so the applied metric needs to comply with the properties of consistency, optimality, and loop-freeness as defined in [48]. Let's assume, $R p$ is a path from the source to the destination node, and $v_{j}^{\prime}$ is the on-path node, then $\varphi\left(R p, v_{j}^{\prime}\right)$ signifies the next-hop of $v_{j}^{\prime}$ on $R p$. In this case, if $v_{j}^{\prime}$ is the destination node of $R p$, then $\varphi\left(R p, v_{j}^{\prime}\right)=N U L L$. Therefore, $\varphi\left(R p, v_{j}^{\prime}\right)$ represents the next-hop on the path to the destination in a multi-hop routing in which $v_{j}^{\prime}$ stores routing information in its table. Each node updates the information when the trickle timer is reset. As explained in [16], the DAG metric container object of DIO message carries the routing metrics and other network information. In the proposed RAROF, the route selection mechanism is represented by a mapping function $\Gamma\left(\left(R p_{i \rightarrow j}\right) \in \mathbb{R}\right)$, which determines the route by calculating the different metric values (i.e., remaining energy, buffer occupancy, ETX, hop count, delay, etc.) of a path between the source node $i$ and the destination node $j$. As a result, each path between nodes $i$ and $j$ has a cost (path weight) that can be ordered, leading to the best path selection, and packets can be routed along the lightest path.

A routing protocol is consistent if data packets from any source node $v_{i} \in V$ to any destination node $v_{j} \in V$ are delivered through any on-path node $v_{j}^{\prime}$ of $\Gamma\left(R p_{i \rightarrow j}\right)$, then other nodes on $\Gamma\left(R p_{i \rightarrow j}\right)$ must take the same decision; i.e., $v_{j}^{\prime}$ forwards packets from $v_{i}$ to $v_{j}^{\prime}$ to $\varphi\left(\Gamma\left(R p_{i \rightarrow j}\right), v_{j}^{\prime}\right)$. According to [49], the routing protocol is known to be optimal if it forwards the data packets through the lightest route (minimum latency, overhead, and energy consumption, etc.) between every pair of nodes in the network. As described in [48,50], the isotonicity and monotonicity characteristics of the routing metric are pertinent to ensure whether a routing protocol can satisfy the consistency, optimality, and loop-free requirements during path construction. Therefore, in this paper, we only consider the isotonicity and monotonicity properties of the routing metrics as follows.

Suppose $\leq$ is used to characterize an order relation, and $\oplus$ is the operator used to concatenate any two routes. The isotonicity of the routing metric states that the order relation among the weights of any two routes is preserved when both routes are prefixed or suffixed by a third common route. It can be expressed mathematically, as follows.

$$
\begin{gathered}
\Gamma\left(R p_{v_{i} \rightarrow v_{j}}\right) \leq \Gamma\left(R p_{v_{k} \rightarrow v_{l}}\right) \Rightarrow\left\{\begin{array}{c}
\Gamma\left(R p_{v_{i} \rightarrow v_{j}} \oplus R p_{v_{m} \rightarrow v_{n}}\right) \leq \Gamma\left(R p_{v_{k} \rightarrow v_{l}} \oplus R p_{v_{m} \rightarrow v_{n}}\right) \\
\Gamma\left(R p_{v_{o} \rightarrow v_{p}} \oplus R p_{v_{i} \rightarrow v_{j}}\right) \leq \Gamma\left(R p_{v_{o} \rightarrow v_{p}} \oplus R p_{v_{k} \rightarrow v_{l}}\right)
\end{array}\right. \\
\forall R p_{v_{i} \rightarrow v_{j}}, R p_{v_{k} \rightarrow v_{l}}, R p_{v_{m} \rightarrow v_{n}}, R p_{v_{o} \rightarrow v_{p}} \in \mathbb{R}
\end{gathered}
$$


On the other hand, the routing metric is monotonic if the path cost or weight does not improve when prefixed or suffixed by another path. Mathematically, it can be expressed as follow.

$$
\left\{\begin{array}{l}
\Gamma\left(R p_{i \rightarrow j}\right) \leq \Gamma\left(R p_{i \rightarrow j} \oplus R p_{j \rightarrow k}\right) \\
\Gamma\left(R p_{i \rightarrow j}\right) \leq \Gamma\left(R p_{k \rightarrow i} \oplus R p_{i \rightarrow j}\right)
\end{array}, \forall R p_{i \rightarrow j}, R p_{j \rightarrow k}, R p_{k \rightarrow i} \in \mathbb{R}\right.
$$

\subsection{The Routing Metrics and RAROF Design}

In the RPL objective function, the use of the routing metric is not only to select the path between source and destination but also to find the optimal route among all the potential routes between them. Therefore, the proposed RAROF considers different routing metrics as the route selection criteria. As explained in [27], different OFs are designed to achieve different application goals that consider the link as well as nodes' characteristics, during the parent selection process. Therefore, we consider both node and link representative routing metrics to create a composite routing metric that RAROF can use to select the best path, and through which a node forwards the data packets to ensure the reliability and energy efficiency. In order to define RPL compliant OF for IIoT networks, in which resource is the key concern, in particular the energy consumption, it is inevitable to include node metric that takes into account the energy consumption tendency and state. To this end, we have used energy-oriented metrics that anticipate the energy vulnerability of nodes and enables making a wise decision on route selection.

The NVI metric is proposed to identify the vulnerable node in terms of remaining energy (life of a node) during path construction. Since the emergency monitoring network needs a seamless operation to ensure reliable data delivery, the NVI metric is reasonable to include in routing decisions that prolong the network lifetime and provide reliability. If the maximum energy capacity and the energy already consumed by a node $v_{i}$ is given by $\operatorname{Ecap}\left(v_{i}\right)$ and $\operatorname{Econ}\left(v_{i}\right)$, respectively, then the NVI can be defined as follows.

$$
\begin{gathered}
\operatorname{NVI}\left(v_{i}\right)=\delta \cdot\left(\frac{\operatorname{Ecap}\left(v_{i}\right)+\operatorname{Econ}\left(v_{i}\right)}{\operatorname{Econ}\left(v_{i}\right)}\right) \times \frac{1}{D c} \\
D c=\frac{S D}{B I}=2^{S O-B O}, \text { such that } 0 \leq S O \leq B O \geq 14
\end{gathered}
$$

where $\delta$ is a constant normalization factor used to normalize the metric value and $D c$ denotes the duty cycle, which is defined as the ratio of time in which the node is in active in SD within a BI. In this work, we define $\operatorname{Ecap}\left(v_{i}\right)$ as the maximum initial energy of a node. In our experiment, the value of $\delta$ is set to 10. According to [51], the appropriate $D c$ can be obtained based on the potential combination between $S O s$ and $B O s$ that satisfies Equation (1). In our proposal, we use the same function to define the duty cycle in the network. The energy consumption tendency of a node typically depends on its position, its role in the network, and other factors. To understand the energy vulnerability, we explicitly take into account the level of energy depletion within the available active duration for communication. Therefore, we ignore the idle state of the node, as radio communication is the dominant energy consumer of a node's battery. Hence, the energy consumed, $\operatorname{Econ}\left(v_{i}\right)$, by a node can be calculated as follows:

$$
\operatorname{Econ}\left(v_{i}\right)=\frac{P c\left(v_{i}\right) \times T_{t r}{ }^{d}\left(v_{i}\right) \cdot \omega}{D_{b}{ }^{r}}
$$

where $P c\left(v_{i}\right)$ is the energy disbursed on each transmitted bit by a node $i, T_{t r}{ }^{d}\left(v_{i}\right)$ is the total data traffic transmitted by a node $i, \emptyset$ is the quality of the link, and $D_{b}{ }^{r}$ is the specified data rate in bits per second;

$$
T_{t r}{ }^{d}\left(v_{i}\right)=T_{t x}\left(v_{i}\right)+\sum_{v_{i-1} \in x} T_{r x}\left(v_{i-1}\right)
$$

where $T_{t x}\left(v_{i}\right)$ is data traffic transmitted by a node itself, $T_{r x}\left(v_{i-1}\right)$ is the data traffic received from all the associated child nodes of a node, $x$ denotes the set of child nodes associated in under single 
hop. In our experiment, we use Energest, an energy estimation module of Contiki OS. This module is used to know how long the operating states (i.e., transmission reception, processing, and low power mode) last. To know the power consumed by these states, we have used CC2420 chipset datasheet [52]. Moreover, we have used Tmote Sky nodes using CC2420, whose Tx and Rx consume almost similar energy per unit time.

Besides, to represent the quality of link $(\omega)$, we condition the ETX metric. ETX is a reliability metric and indicates the expected number of transmissions that a node required for successful data packet delivery to the destination. Suppose $l_{i \rightarrow j}$ is the link between two nodes $i$ and $j$, then $l q(i, j)$ represents the ETX metric in a path connecting node $v_{i}$ to node $v_{j}$ in $\mathbb{R}$. Therefore, the link quality metric, $l q$, is an additive when it adds ETX of each link in the path to the destination node as follows:

$$
l q\left(R p\left(v_{i}, v_{j}\right)\right)= \begin{cases}l q\left(v_{i}, v_{i+1}\right)+l q\left(v_{i+1}, v_{i+2}\right)+l q\left(v_{i+2}, v_{i+3}\right)+\ldots+l q\left(v_{i+m}, v_{j}\right) & \text { if } \varphi\left(R p, v_{i+1}\right) \neq N U L L \\ l q\left(v_{i}, v_{j}\right) \text { if } \varphi\left(R p, v_{i+1}\right)=N U L L\end{cases}
$$

The link quality increases as the value of $l q$ decreases, so the metric value near one indicates the optimum communication link. Consequently, the best $l q$ metric helps in the optimal path selection, which eventually contributes to prolonging the network lifetime. The advantage of using this metric during the RPL route selection process is to avoid links with poor communication quality. In our proposal, at each node, the $l q$ metric is measured as in Equation (7).

$$
l q\left(v_{i}\right)=\alpha \times l q_{\text {old }}+(1-\alpha) \times l q_{\text {recent }}
$$

where $l q_{\text {old }}$ is the prior average ETX for a node and stores this value in its routing table, $l q_{\text {recent }}$ is the recent measured ETX, which can be obtained as in Equation (8), and $\alpha$ is the learning ratio such that $0<\alpha<1$. We have set $\alpha=0.9$, according to the Contiki RPL for the better smoothing effect.

$$
l q_{\text {recent }}=\frac{1}{d_{i \rightarrow j} \times d_{j \rightarrow i}}
$$

where $d_{i \rightarrow j}$ is the probability that the data packet successfully delivered to the recipient node and $d_{j \rightarrow i}$ is the probability that the acknowledgment is successfully received by the sender node.

The other metric used in our proposal is the resource availability, $R_{a v}$, which indicates the available resource in terms of the buffer capacity availability in the node. To this end, we have mapped $R_{a v}$ with the binary parameters 0 and 1, as shown in Equation (9), which indicates either the resource in a node is available or not.

$$
R_{a v}\left(v_{i}\right)=\left\{\begin{array}{c}
1 \text { if }\left(1-\frac{B_{a v}\left(v_{i}\right)}{T_{b u f}\left(v_{i}\right)}\right)^{2}<\tau^{t h} \\
0 \text { otherwise }
\end{array}\right.
$$

where $B_{a v}$ is the available buffer space of a node and $T_{b u f}$ is the total buffer size of node $i . \tau^{\text {th }}$ is a predefined buffer threshold. For our experiment, we use a static threshold and set to $85 \%$ of the total size of the queue of a node. When the buffer utilization of a node is higher than the threshold $\tau^{\text {th }}$, the resource availability set to zero, and the RAROF ignores such nodes during the route selection process.

Now, the proposed RAROF dynamically select the routing path based on the routing metrics defined above. Like the default RPL, in this implementation, RAROF performs globally throughout the network. In the RPL scope, a node construct the routing path to the DODAG root by selecting the preferred parent from the candidate parent set. Let assume that for each node $v_{i} \in V(i=1,2,3, \ldots, n)$, $N B(i) \subseteq V$ is the subset of all eligible neighbor nodes that can communicate with each other. Each node $i$ maintains its neighbor table and adds a new node $j \in N B(i)$, only if there is space in the table to store. Afterward, each node $v_{i} \in V$ creates a candidate parent set $C p\left(v_{i}\right)=\left\{p_{1}, p_{2}, p_{3}, \ldots, p_{n}\right\}$ from its neighbor table, from which a node chooses its next-hop toward the DODAG root, i.e., preferred parent 
$P p\left(v_{i}\right)$ and establishing the routing path to forward the data packets. In this entire process, a node $v_{j}$ can be a candidate parent node of node $v_{i}$, only if it satisfies the following conditions.

$$
p \in N B\left(v_{i}\right) ; v_{j} \in N B(p) ; r(p)<r\left(v_{j}\right)
$$

where $r$ denotes the rank of a node. Unlike default RPL implementation [16], in this paper, the preferred parent selection and the rank calculation is detached. Thus, RAROF constructs the DODAG tree structure based on the node's rank, for which the ETX metric is used, and the rank of a node $r\left(v_{i}\right)$ is computed as in Equation (11).

$$
r\left(v_{i}\right)=\min _{j \in N B(i)}\left(l q\left(v_{i}, v_{j}\right)+r\left(P p\left(v_{i}\right)\right)+\gamma\right.
$$

Here, $r\left(P p\left(v_{i}\right)\right)$ is the rank value of the preferred parent of the node $v_{i}$, and $\gamma$ is the constant integer used to confirm the loop-freeness in the network and satisfying the Equation (10). It is defined to ensure the rank increment of a node that satisfies the rank policy of the DODAG-tree structure specified in [16]. Finally, the proposed RAROF selects the next-hop node based on the minimum function and determines the data forwarding path as follows.

$$
\begin{gathered}
\min \Gamma N V I\left(v_{i}\right)+R_{a v} \\
\text { s.t. } R_{a v}=1
\end{gathered}
$$

Furthermore, the proposed metric also meets the primitive requirements such as isotonicity and monotonicity, the proof is as follows.

Let us assume that there exist $n$ routing paths $R p_{n} \in \mathbb{R}$, where $n=3$. These three routing paths are represented as $R p_{1}=\mathbb{R}\left(v_{i}, v_{j}\right)=\left(v_{i}, v_{i+1}, v_{i+2}, v_{i+3}, \ldots, v_{j}\right), R p_{2}=\mathbb{R}\left(v_{i}^{\prime}, v_{j}^{\prime}\right)=\left(v_{i}^{\prime}, v_{i+1}^{\prime}, v_{i+2}^{\prime}, v_{i+3}^{\prime}, \ldots, v_{j}^{\prime}\right)$, and $R p_{3}=\mathbb{R}\left(v_{i}^{\prime \prime}, v_{j}^{\prime \prime}\right)=\left(v_{i}^{\prime \prime}, v_{i+1}^{\prime \prime}, v_{i+2}^{\prime \prime}, v_{i+3^{\prime}}^{\prime \prime}, \ldots, v_{j}^{\prime \prime}\right)$. Next, the metric values of these paths are as follows.

$$
\begin{gathered}
\Gamma\left(N V I\left(R p_{1}\right)\right)=\Gamma\left(N V I\left(v_{i}, v_{i+1}\right) \oplus N V I\left(v_{i}, v_{i+1}\right) \oplus \ldots \oplus N V I\left(v_{j-1}, v_{j}\right)\right)=\sum_{x=1}^{n} \Gamma N V I\left(v_{x}\right) \geq 0 \\
\Gamma\left(N V I\left(R p_{2}\right)\right)=\Gamma\left(N V I\left(v_{i}^{\prime}, v_{i+1}^{\prime}\right) \oplus N V I\left(v_{i}^{\prime}, v_{i+1}^{\prime}\right) \oplus \ldots \oplus N V I\left(v_{j-1}, v_{j}\right)\right)=\sum_{x=1}^{n} \Gamma N V I\left(v_{x}^{\prime}\right) \geq 0 \\
\Gamma\left(N V I\left(R p_{3}\right)\right)=\Gamma\left(N V I\left(v_{i}^{\prime \prime}, v_{i+1}^{\prime \prime}\right) \oplus N V I\left(v_{i}^{\prime \prime}, v_{i+1}^{\prime \prime}\right) \oplus \ldots \oplus N V I\left(v_{j-1}^{\prime \prime}, v_{j}^{\prime \prime}\right)\right)=\sum_{x=1}^{n} \Gamma N V I\left(v_{x}^{\prime \prime}\right) \geq 0
\end{gathered}
$$

Here, $x$ is the traversed node in $R p_{n} \in \mathbb{R}$. The metric value of each routing path is always $\geq 0$. If the path function $\Gamma N V I\left(R p_{1}\right) \leq \Gamma N V I\left(R p_{3}\right)$, then $\Gamma N V I\left(R p_{1}\right)+\Gamma N V I\left(R p_{2}\right) \leq \Gamma N V I\left(R p_{3}\right)+\Gamma N V I\left(R p_{2}\right)$ can be easily proved, regardless of the prefixed or suffixed by third common path such as $R p_{2}$. Therefore, it is proved that the proposed metric satisfies the isotonicity requirement. On the other hand, the metric value of each routing path is always $\geq 0$, so, $\Gamma N V I\left(R p_{1}\right) \leq \Gamma N V I\left(R P_{1}\right)+\Gamma N V I\left(R p_{2}\right)$ can be easily proved regardless of the prefixed or suffixed by a third common path such as $R p_{2}$. In this way, it also proves that the proposed metric meets the monotonic requirement.

\subsection{Multi-Queuing Scheme for Efficient Data Delivery}

Aiming to ensure efficient data delivery for IEMS, in our proposal, we adopt the queuing model presented in [53] to handle the critical and non-critical traffic types. To achieve high reliability and lower delay when routing critical data traffic, reliability sensitive packets should be given high priority as compared to regular packets. Hence, this priority can be specified through a multi-queuing system, which is controlled by each node. Consequently, the implementation of a multi-queuing strategy allows the prioritization of data traffic to ensure high reliability to critical packets. In our priority queuing approach, we use two different queues, one for critical traffic, and the other abide non-critical traffic (i.e., regular traffic). The queuing model sends packets from the highest priority queue first, 
followed by the lowest priority queue. The high priority queue is referred to as a critical traffic queue (CTQ), which is used by critical packets, the low priority queue is denoted as regular traffic queue (RTQ), which is used by the non-critical packets or regular packets. We undertake that each source node is aware of the importance of each data packet it is transmitting that can be translated into a predetermined priority level. For the IEMS use case of IIoT, the application layer defines the priority level of each data packet generated by adding extra information to differentiate critical and non-critical packets. Subsequently, the classifier routes the packets into the appropriate queue based on the packet type. In the emergency monitoring network, when an unusual situation occurs, the node generates data for the appropriate action; therefore, the number of critical packets in the queue would be relatively low compared to regular packets, and for some time extent, the queue could be empty. Meanwhile, lower priority traffic may be obstructed by high priority traffic indefinitely. To overcome this situation, we use the timeout policy for each packet, so that contention priorities can be specified explicitly between packets from the same node. Algorithm 1 describes the implementation of the multi-queuing strategy in this paper.

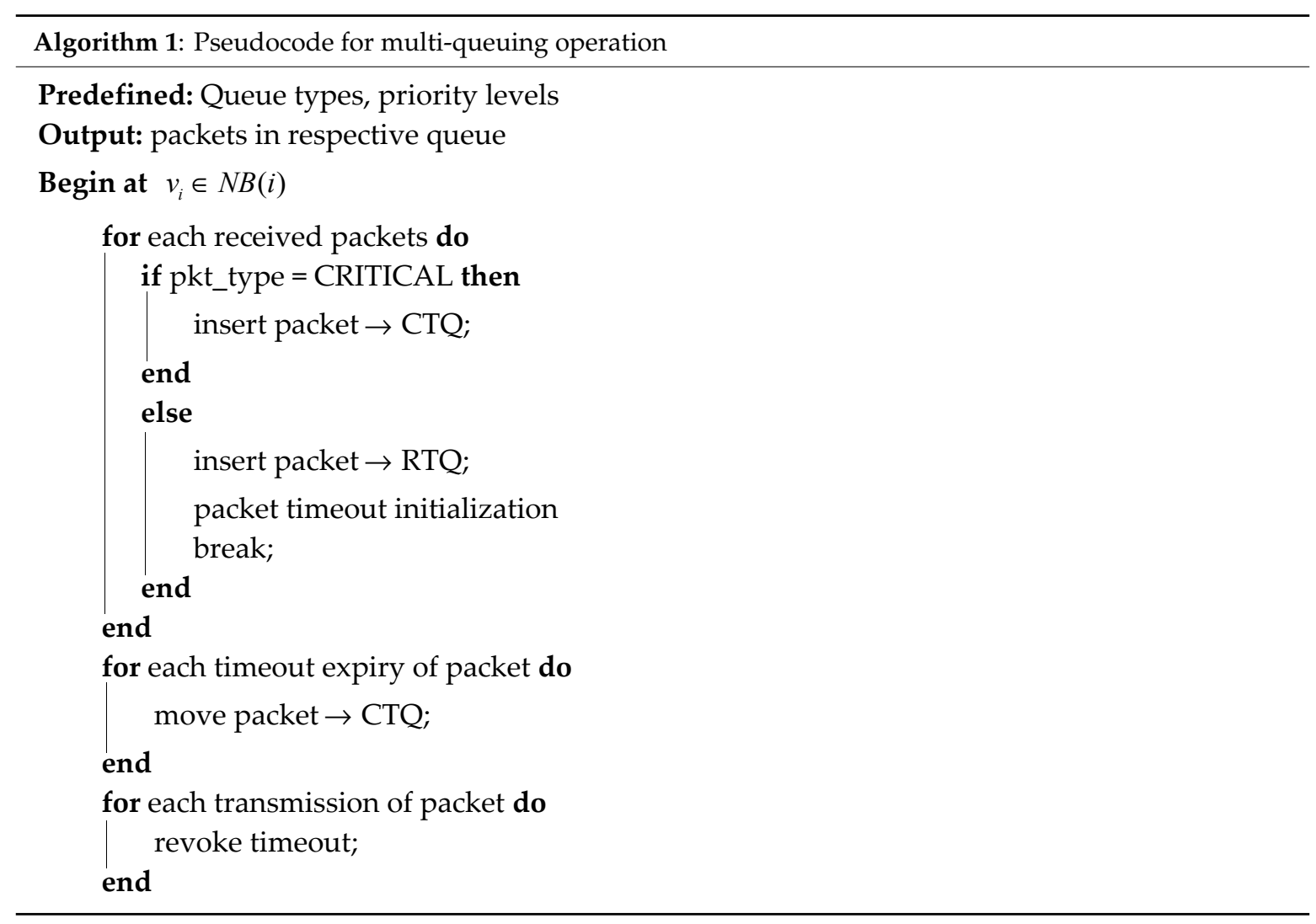

\subsection{Conceptual Cloud-Assisted IEMS Architecture for RPL Based IIoT Network}

The idea behind the IIoT is to break down technical barriers and facilitate the integration of the physical and digital worlds. The promising potential of the cloud services has played a significant role in the feasibility of the IIoT. Because of the sensitive and critical ambient in the industries, the real-time monitoring system is an important aspect for analyzing data to enhance the proficiency, service quality, and providing an immediate response. IEMS is a combination of applications, people, interconnected things such as sensors, actuators, embedded devices, etc., and state of art technologies, which would function together to collect, monitor, track, control, store, and share information for better results. In this section, we present the theoretical IEMS design that provides the emergency monitoring framework, to which the surrounding and other functioning data are collected and sent to the cloud 
for analysis and evaluation, which enables the seamless access to authorities, engineers, and operators. The cloud-assisted IEMS architecture is as shown in Figure 2.

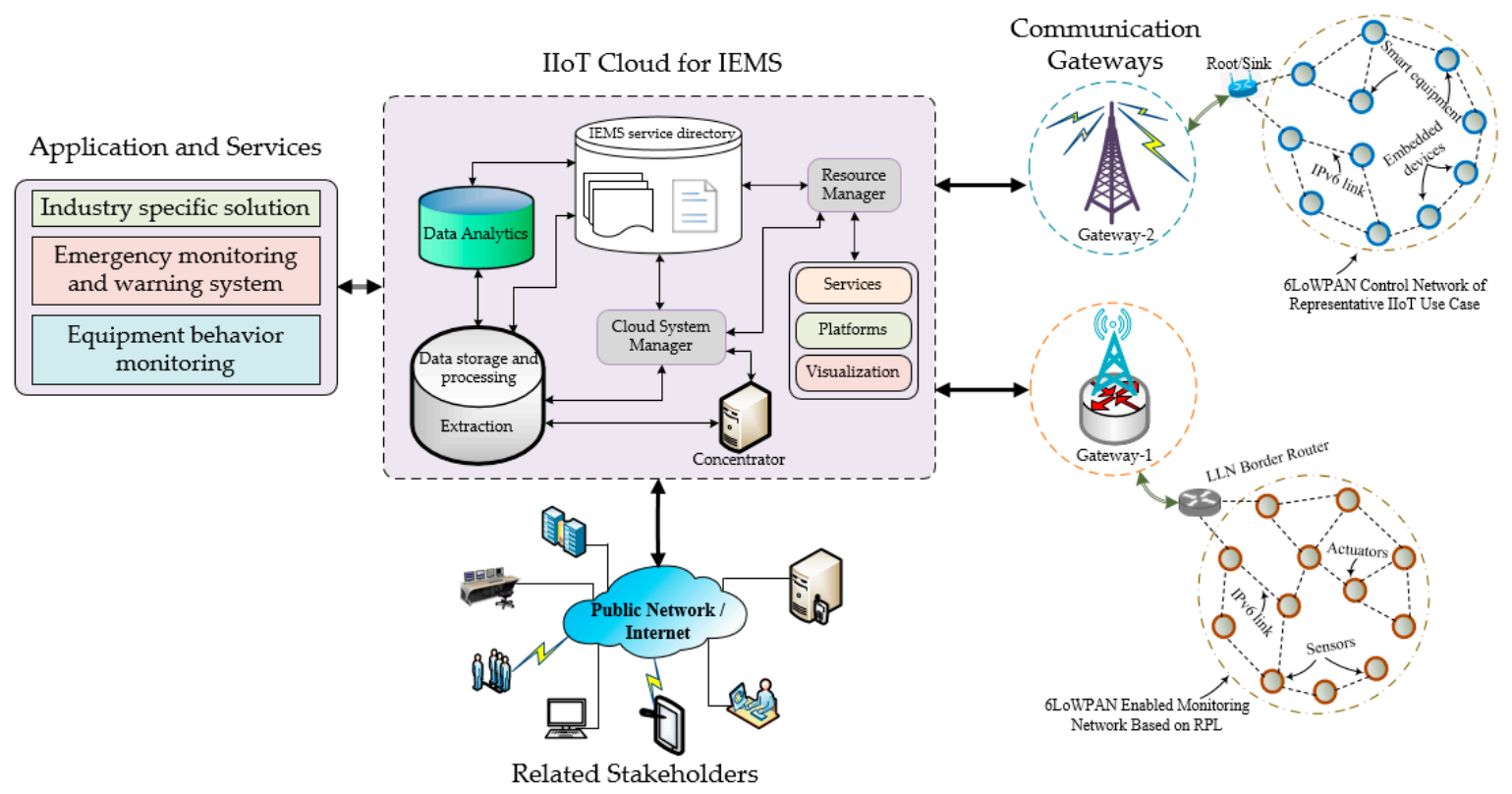

Figure 2. Cloud-Assisted Industrial Emergency Monitoring System (IEMS) architecture.

The architecture outlines how the flow of the IEMS data is captured and seamlessly forwarded to the IIoT cloud center via a communication gateway for further analysis and processing. Later, the processed information is either assessed and used by engineers, operators, paramedics, and control center or delivered to the exterior system for a specific solution. The main components in this architecture are as follows.

Connected Network: Data acquisition is performed by different sensors, embedded devices, equipment, and actuators through connection with an intermediate data aggregator. Using the network connection, the data aggregator sends the information to the cloud system where the concentrator extract some features, classify them, and process further.

Resource Manager: Assigns the resources to virtual machines as required. It manages services that fulfill user requirements. Moreover, it provides a cost-efficient platform to IEMS IIoT.

Data Transmission: It is responsible for conveying the acquired data to the cloud system, typically in real-time with the assured security and privacy. Generally, the sensing layer is equipped with a low-power and short-range radio such as low-power Bluetooth, IEEE 802.15.4, or ZigBee.

Individuals and Related Stakeholders: After data processing and analysis in the cloud system, the surrounding and other operational records are stored in the database, in which professionals and other stakeholders can assess and review it for further action.

Cloud System Manager: Defines and assigns the appropriate resources via resource manager and controls all the virtual machines for different services. In addition, it provides the IEMS service interaction method to application and services through a service directory.

Data Storage and Processing: It stores the aggregated sensory records and other information to be used for the long term. Data storage to the cloud system offers the benefits of providing scalability and accessibility on request for both users and other stakeholders. Furthermore, cloud processing can decrease costs and provide better real-time information.

IEMS Service Directory: It registers and accumulates data from the sensing regions. Besides, it records and issues multiple services that take part in the IEMS. Some of the typical services in this directory are alert notification, predictive maintenance, and relief and rescue classification.

The steady and uninterrupted nature of various services in the industries has challenged traditional monitoring ways and means. In recent years, sophisticated techniques for monitoring and analyzing the 
situation has evolved due to the advancement of technology, smart devices, and tiny chips. Therefore, it is clear that the IIoT plays a significant role in the next-generation emergency monitoring system.

\section{Experimental Results and Evaluation}

In this section, we present the performance evaluation of our proposed routing scheme based on RAROF by using simulation. Furthermore, we describe the simulation environment, simulation parameters, and depict the comparative simulation results. The extensive simulation experiments are performed in the COOJA simulator and compared with other OFs to validate the proposed OF in the IIoT scope.

\subsection{Simulation Environment and Parameters}

In our simulation study, we have used topology with two aggregators (i.e., DODAG root) and 140 smart devices (i.e., sensor nodes), randomly scattered in the area of $300 \mathrm{~m} \times 300 \mathrm{~m}$. The DODAG roots were placed at the center of the simulation area, and other nodes were spread over the sensing area. In the network, the nodes communicate with each other either directly or in a hop-by-hop manner. In our simulated scenario, all the nodes were configured as Tmote Sky motes with CC2420 wireless transceiver. We considered that the network generates frames of different sizes at varying intervals. Therefore, the maximum size of each data packet was restricted to 127 bytes, and data rate to $250 \mathrm{kbps}$. The total time for each simulation run was $1800 \mathrm{~s}$. We performed our simulation 10 times for each test and drawn the average results to minimize the errors. The trickle timer parameters are set to the default value. In the graphical description, the error bar shows $95 \%$ confidence intervals. Table 1 shows the remaining key simulation parameters of our experiment.

Table 1. Simulation parameters for our experiment.

\begin{tabular}{cc}
\hline Parameters & Value \\
\hline Operating system & Contiki 3.0 \\
Radio model & Unit Disk Graph Medium (UDGM) \\
Traffic rate & CBR flow \\
Energy consumption & CC2420 based \\
MAC protocol & Contiki MAC \\
Transport & UDP/Ipv6 \\
Max queue size & 30 packets \\
Transmission power & $-17 \mathrm{dBm}$ \\
Initial energy of each node & $10 \mathrm{Joule}$ \\
Channel check rate & $16 \mathrm{~Hz}$ \\
Routing protocol & $\mathrm{RPL}$ \\
Traffic rate & $15,30,45,60,(\mathrm{ppm})$ \\
Max retransmission & 3 \\
$\gamma$ & 1 \\
\hline
\end{tabular}

\subsection{Performance Metrics}

To compare and analyze the performance of the routing techniques with other OFs available, we have defined the following qualitative performance metrics.

Packet delivery ratio (PDR): This is the ratio of the number of the data packet received at the root node and the total number of packets sent by the source nodes, which is as follows:

$$
\begin{gathered}
\text { PDR }=\frac{\# \text { of packets successfully received at root node }}{\text { \# of packets sent }} \\
\# \text { of pacekts sent }=\text { \# of packets sent by each node } \times \sum_{v_{i}=1}^{n} \# \text { of nodes }
\end{gathered}
$$


Network lifetime: We considered it as the time to network partition. Network partition occurs when the average number of nodes dead in the network. Similar to [54], in our evaluation, we use it as a metric that will use energy variance, which can be defined as follows.

$$
\begin{aligned}
\text { Network lifetime } & =I_{e}-\left(\bar{U}_{e}+\varsigma\right), \\
\bar{U}_{e} & =\frac{\sum U_{e}}{N}
\end{aligned}
$$

where $I_{e}$ is the initial energy of a node, $U_{e}$ is the average used energy, and $N$ is the total number of nodes in the network. The energy is calculated based on the power consumed by the node in each of the states such as Tx, Rx, idle, and CPU (radio is off, but the system is active while processing) in which a node can function. Now, $\varsigma$ can be expressed as follows:

$$
\varsigma^{2}=\frac{\left(U_{e}-\bar{U}_{e}\right)}{N}
$$

Number of nodes alive: This is known as the number of active nodes in the network that can operate. In our analysis, we have considered the node to be alive, whose remaining energy is more than $2 \%$ of its initial energy.

Energy cost per data received: This is defined as the average energy consumed for a successful data packet delivered to the destination node.

\subsection{Simulation Results and Discussion}

The performance of OF-EC, OF-ER, and RAROF are intensively evaluated in various aspects. We first examine the impact of transmission range for all OFs in terms of energy efficiency, lifetime, and reliability. Figure 3 a shows the energy expenditure of a successfully delivered data packet at the destination by OF-EC, OF-ER, and RAROF with respect to the varied transmission range. As the transmission range increases, the energy consumption of each OF decreases accordingly. It is clear that the reduced energy consumption can be achieved with RAROF compared to other OFs. This is because, the higher transmission range helps each node making more successful data transmission, which reduces the number of lossy links that a node should traverse to reach its destination. Besides, the traffic differentiation through the multi-queuing technique allows the node to prioritize the data packet according to the traffic types. Furthermore, the RAROF adopts the duty cycle information in a routing process, which enables nodes aware of the active and inactive status. In this manner, the possibility of re-transmission is reduced, which results in an energy cost reduction. As compared to OF-EC, OF-ER have achieved better energy cost because it considers various metric while selecting parent such as queue, delay, link quality, etc.

From Figure 3b, we notice that for the varied transmission capacity, RAROF achieves the higher numbers of alive nodes in the network compared to OF-ER and OF-EC. When the transmission range of each node is $60 \mathrm{~m}$, RAROF outperforms OF-ER and OF-EC by achieving about $11 \%$ and $21 \%$ of more alive nodes, respectively. On the other hand, when the transmission range is $20 \mathrm{~m}$, the performance is very similar. Therefore, we consider $60 \mathrm{~m}$ as default for our simulation experiments.

Figure $3 c$ shows the packet delivery ratio of OF-EC, OF-ER, and RAROF in percentage under varying transmission range. The result depicts that with the increasing transmission range, the packet delivery ratio also increases for all OFs. Nevertheless, RAROF obtains the higher PDR than OF-EC and OF-ER. Compared to others, RAROF significantly achieves more alive nodes in the network, which provides an opportunity to find the optimal routing path, resulting in the higher PDR. Besides, it adopts a multi-queuing approach for traffic differentiation, and data packets are forwarded, to the proper channel (i.e., fixed sized slots or reserved slots), according to the traffic priority. Thus, RAROF can maintain its reliability of a maximum of $86.7 \%$ and a minimum of $32.5 \%$ when the transmission range is 60 and $20 \mathrm{~m}$, respectively. In contrast, OF-ER improves its reliability by $15.4 \%$ and $4.5 \%$ higher than OF-EC when the transmission range is 60 and $20 \mathrm{~m}$, respectively. It was 
expected because OF-EC is not aware of its resources in terms of queue utilization and the number of overburden nodes. On the other hand, at the transmission range of $60 \mathrm{~m}$, OF-EC maintains the reliability of a maximum of $56.7 \%$, which is about $30 \%$ less than RAROF, and it is not acceptable for emergency monitoring application of IIoT. Besides, during parent selection, both OF-ER and OF-EC do not allow to be aware of nodes' sleep and awake states, which leads to higher packet retransmission, eventually affecting the network reliability.

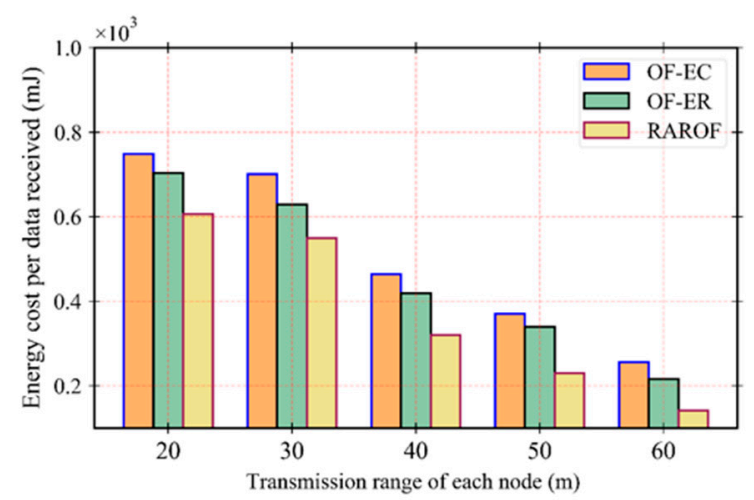

(a)

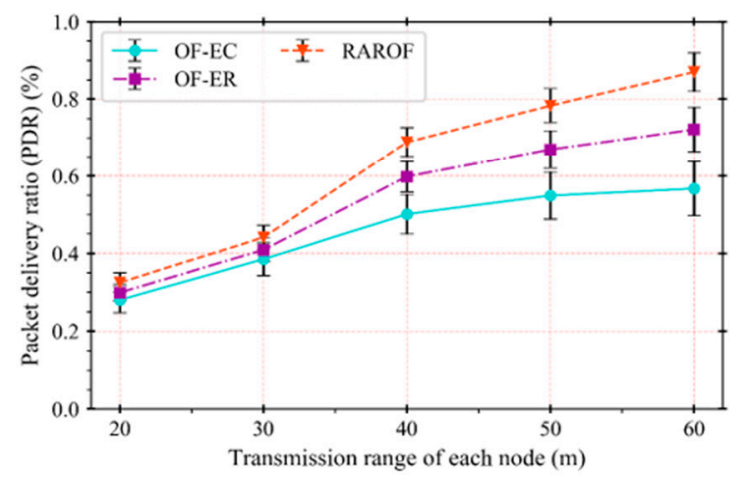

(c)

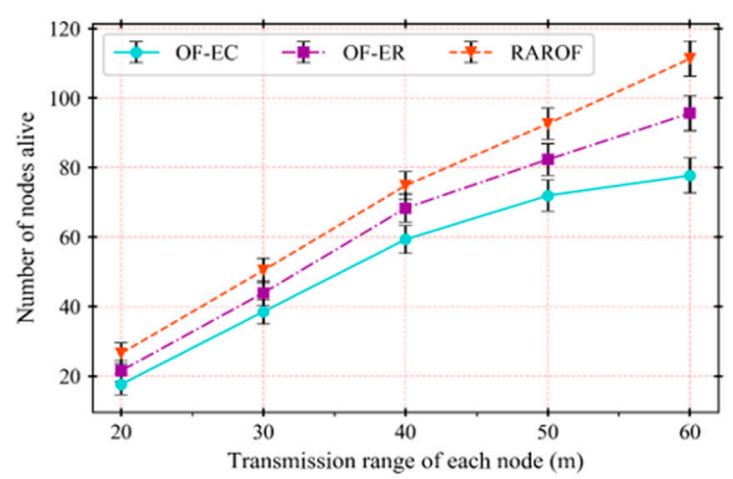

(b)

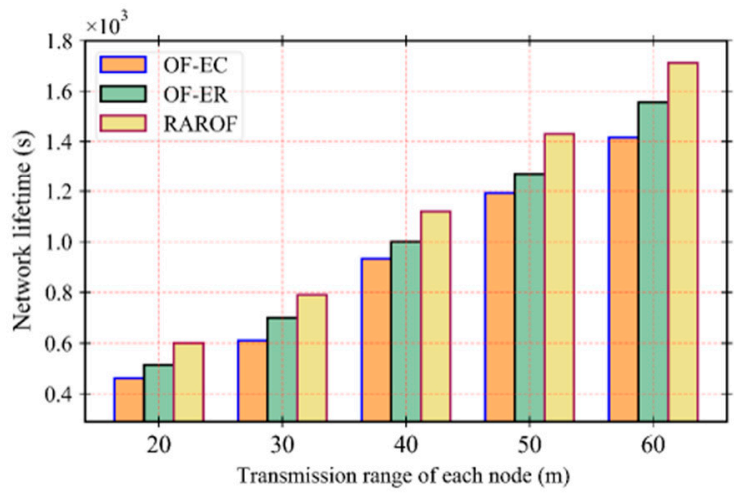

(d)

Figure 3. Impact of transmission range; (a) energy cost per data received, (b) number of nodes alive in the network, (c) packet delivery ratio (PDR) in percentage, and (d) network lifetime in seconds.

As can be seen in Figure 3d, with the increasing transmission range, the network lifetime also increases consistently. The result reveals that RAROF can effectively delay the occurrence of the network partition as compared to OF-ER and OF-EC. When the transmission range is 20 and $60 \mathrm{~m}$, RAROF shows the superiority in the network lifetime performance than OF-ER and OF-EC by about $5.2 \%$ and $8.7 \%$ and $8.2 \%$ and $16.3 \%$, respectively. This is because the routing mechanism of RAROF can effectively reduce the interruption of communication due to network partition. Besides, RAROF makes use of network resources effectively during the route construction process, resulting in more numbers of alive nodes. Consequently, it prolongs the network lifetime as compared to others. On the other hand, as compared to OF-EC, OF-ER provides a better network lifetime because it also considers the resources during the parent selection process, however, it does not include the radio duty cycle, which is one of the key aspects of evaluating the energy consumption of a node. Moreover, the result shows that a multi-queuing based traffic differentiation scheme in RAROF can prolong the network lifetime. This is because data packets are prioritized to access the channel, through which the tendency of packet drop reduces significantly, consequently decreases packet retransmission and saves the node's energy. To obtain high reliability when routing critical data packets, higher priority is given to these packets in channel contention than the regular packets. For this, a node uses its reserved slots for critical data packets and contention access period for regular data packets. Due to the prioritization, 
RAROF not only achieves the higher PDR but also increases the network lifetime by reducing the packet retransmission.

Afterward, we examined the impact of the varying traffic load on RAROF performance. To this end, we set the transmission range of each node to $60 \mathrm{~m}$. To study the network performance in terms of energy efficiency, reliability, and lifetime, the varied traffic load is offered from 15 to 60 packets per minute. In Figure $4 a-d$, we have presented the performance of OFs considering different traffic loads. Figure 4a illustrates that the network with higher traffic load has increased energy consumption while delivering data successfully to the destination. The great deal of energy expenses is conserved by RAROF during data delivery under high traffic load. Whereas, OF-EC and OF-ER have comparatively consumed higher energy for successfully delivering data packets. In Figure $4 \mathrm{~b}$, it can be seen that as the traffic load increases, the number of alive nodes decreases significantly for all OFs. However, as compared to others, RAROF obtains a higher number of alive nodes in the network. After these analyses, we examined the reliability of the network under different traffic load.

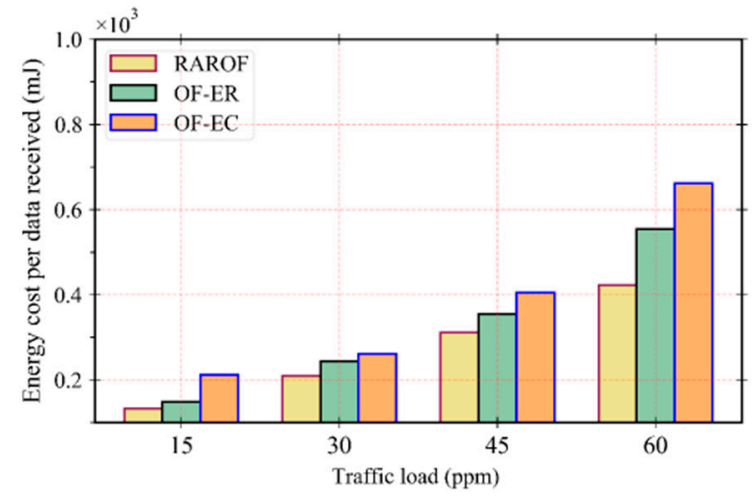

(a)

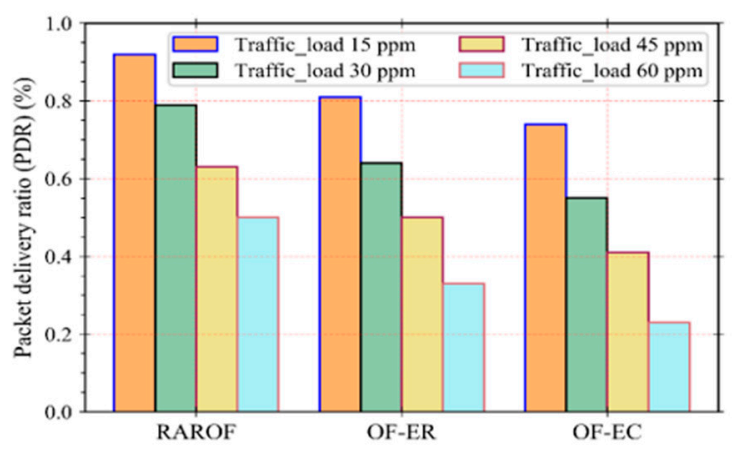

(c)

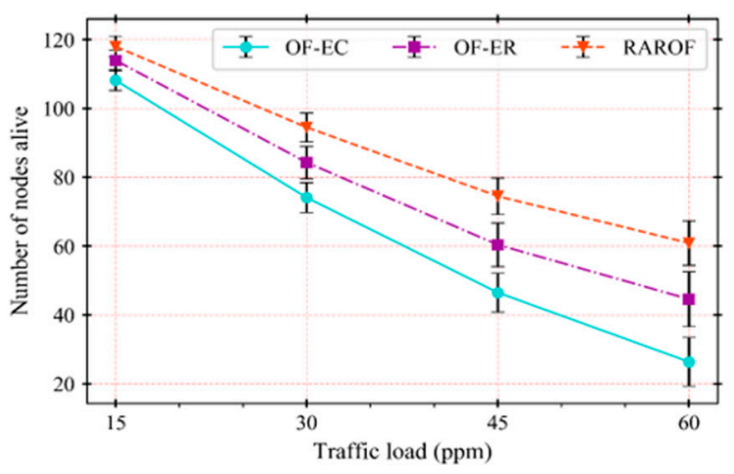

(b)

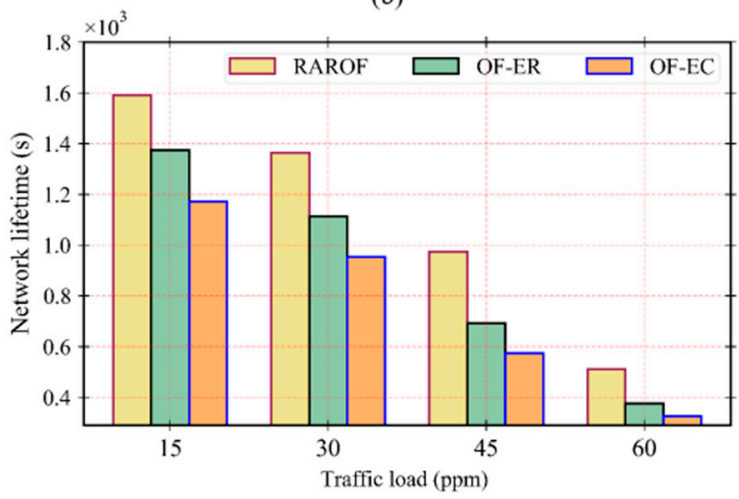

(d)

Figure 4. Impact of traffic load; (a) energy cost per data received, (b) number of nodes alive in the network, (c) PDR in percentage, and (d) network lifetime in seconds.

Figure 4c shows that as the traffic load increases, the packet delivery ratio of RAROF, OF-ER, and OF-EC decreases. This tendency can be described by the fact that under heavy traffic flow, generated and received data packets often backlogged at the node's buffer, which leads to more retransmission and packet loss, resulting in a lower PDR. However, in this regard, RAROF prioritizes the traffic types and differentiate them using a multi-queuing system, as a result, achieves higher PDR as compared to others. Moreover, RAROF not only differentiates the traffic type but also aware of the node's buffer utilization during route construction. Therefore, it achieves a minimum and maximum reliability of $44 \%$ and $86 \%$, respectively, when the traffic load is 60 and $15 \mathrm{ppm}$. On the other hand, OF-ER maintains higher reliability than OF-EC, one of the main reasons for this achievement is that it includes the node's queue utilization metric during route construction. From Figure $4 \mathrm{~d}$, we can observe that the network lifetime decreases constantly for all OFs with the increased traffic load. The network 
topology constructed under RAROF alleviates partition for a long time as compared to OF-ER and OF-EC. This indicates that RAROF can delay the death of a node in the network due to the node's energy consumption.

After these assessments, we analyzed the effect of the reporting interval on network partition. In this regard, we utilized various reporting intervals ranging from 2 to $8 \mathrm{~s}$. It is define as the time interval between the arrival of data packets. We set the transmission range of each node to $60 \mathrm{~m}$. Figure 5a shows that RAROF can keep the nodes alive to a higher number as compared to other OFs. It also illustrates that as the reporting interval increases, so does the number of active nodes in the network. When the interval is $2 \mathrm{~s}$, the number of alive nodes of OF-EC, OF-ER, and RAROF are almost the same, because the low inter-packet interval leads to higher energy consumption. However, in this situation, RAROF obtains $4.2 \%$ and $5.9 \%$ more alive nodes than OF-ER and OF-EC, respectively. Then, we examined the node's lifespan over time. The number of nodes alive in the network is shown in Figure 5b. From the result, we can observe that the alive nodes in the network decrease for all OFs over time. Nevertheless, RAROF comparatively achieves more alive nodes during simulation time. OF-EC, on the other hand, achieves the lowest number of active nodes over time.

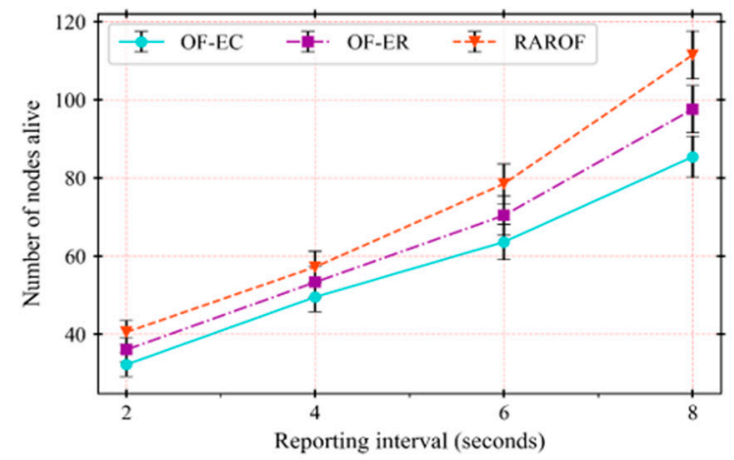

(a)

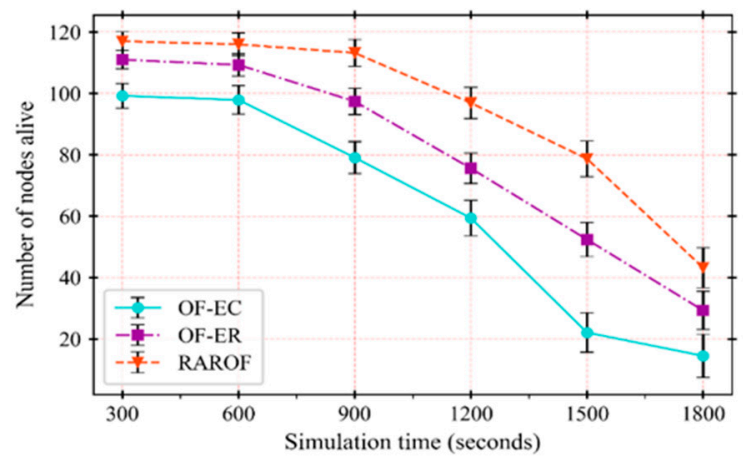

(b)

Figure 5. Nodes alive in the network; (a) number of nodes alive in terms of reporting interval, (b) number of nodes alive over time.

Similarly, we examine the network lifetime in terms of the varied reporting interval. Figure 6a illustrates the lifetime of the network with respect to the reporting interval. The result depicts that with the increasing time interval, the lifetime of the network also increases significantly. This is because the higher the intervals between packets, the lesser the node's buffer filled, resulting in lower packet loss due to buffer overflow, which eventually reduces packet re-transmission and thereby saves energy. At each reporting interval, the network lifetime in RAROF increases, which shows that RAROF can effectively reduce the death of nodes due to energy-constrained. After that, we analyzed the network lifetime over time. Figure $6 \mathrm{~b}$ shows that for each OFs, the network lifetime decreases over time. From the result, it is clear that the network lifetime of RAROF is higher than OF-EC and OF-ER. Moreover, it can be noticed that the occurrence of a network partition is gradually rising with time, and for all OFs, the energy-constrained is negligible at the initial stage of network operation is low. However, OF-EC is most energy vulnerable as compared to OF-ER and RAROF.

Next, we examined the overhead comparison of the OFs. For this, we only considered the overhead of the DIO control messages of each node. In this simulation experiment, we set the traffic load to $15 \mathrm{ppm}$, and the transmission range of each node set to $60 \mathrm{~m}$. We look into more detail about the overhead of route construction and topology management through Figure 7a-d. Therefore, we take into account the traffic load and transmission range to analyze the overhead. Figure 7a shows the average parent change per node vs traffic load. In a resource-constrained network, frequent parent change also has an impact on energy consumption. Consequently, the network lifetime may be affected. However, the average parent changes in RAROF is lower than others. It indicates that RAROF selects a reliable next-hop node than OF-ER and OF-EC. This is because RAROF selects the parent based 
on the minimum function of the NVI metric. Besides, RAROF can be aware of the node's resources availability during parent selection. Likewise, Figure $7 \mathrm{~b}$ shows that RAROF generates a minimum number of control packet even when the traffic load becomes heavy. When the traffic load is high, OF-EC incurs comparatively more routing overhead than RAROF and OF-ER.

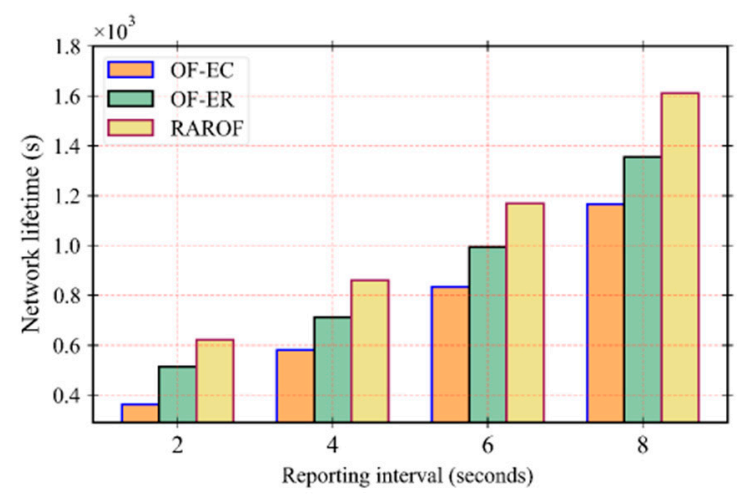

(a)

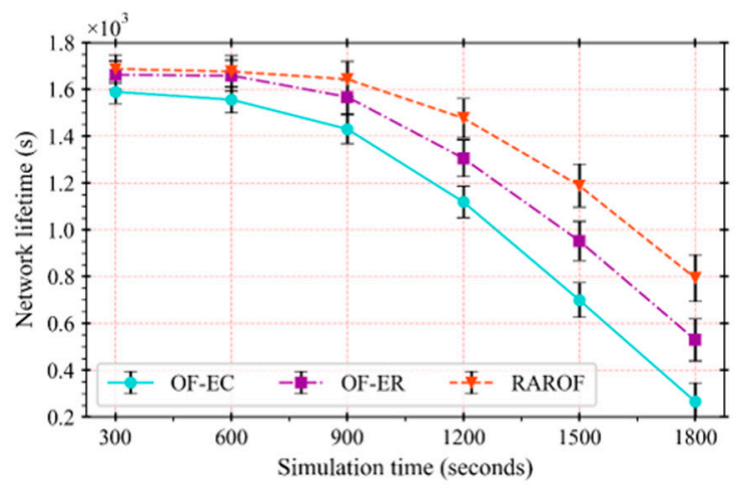

(b)

Figure 6. Lifetime of the network; (a) lifetime in terms of reporting interval, (b) lifetime over time.

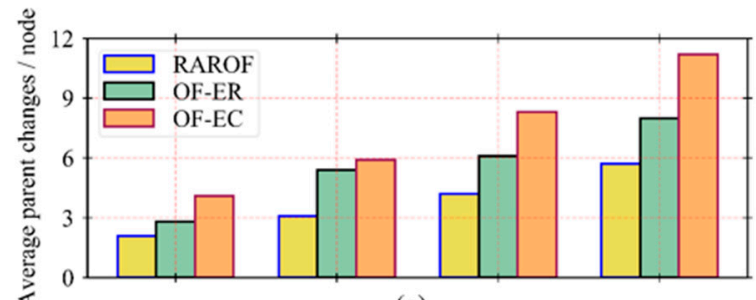

(a)

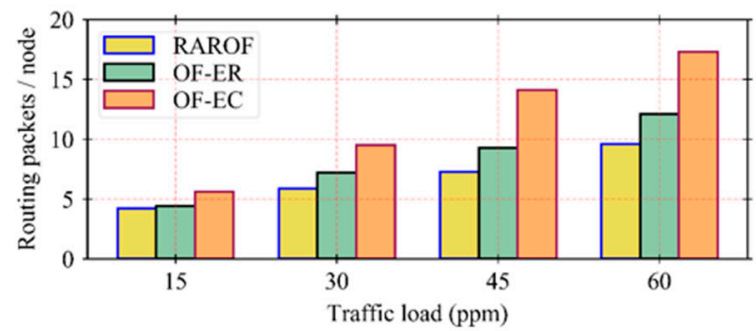

(b)

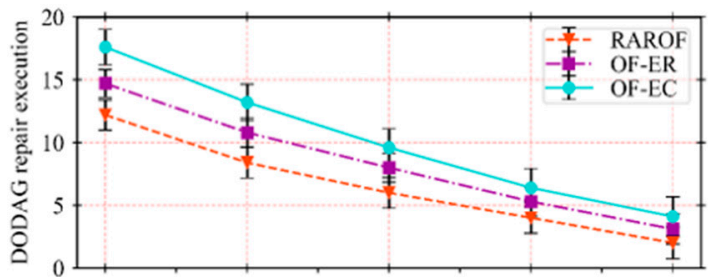

(c)

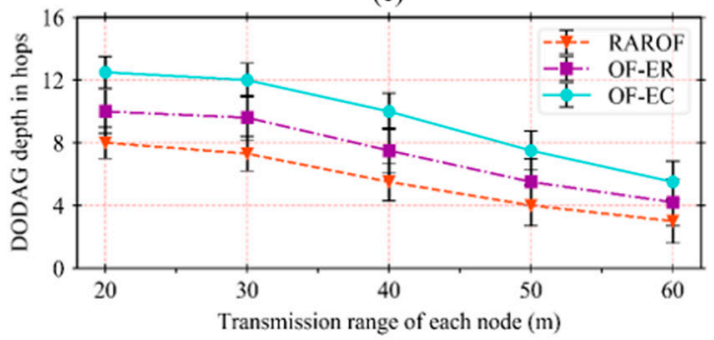

(d)

Figure 7. Overhead comparison of the objective functions (OFs); (a) average parent changes per node, (b) routing packets overhead, (c) destination orientated directed acyclic graph (DODAG) repair execution in the network, and (d) DODAG depth in hops.

Furthermore, we have investigated the different aspect of topology management with varied transmission range. To this end, we consider the DODAG repair execution and DODAG depth as an evaluation criterion. The DODAG repair execution is the topology repair frequency performed by the DODAG root, and DODAG depth is the number of hops from DODAG root. From Figure 7c, it can be seen that OF-EC frequently triggers DODAG repair compared to OF-ER and RAROF. It can be seen that there is a correlation between DODAG repair and routing overhead because when the route failure detects, each node reset the trickle timer for DODAG construction, which leads to a large number of DIO message generation. However, in both cases, RAROF outperforms OF-ER and OF-EC, and hence enhance energy efficiency. Similarly, Figure $7 \mathrm{~d}$ reveals that RAROF constructs topology with lower hop distance than OF-ER and OF-EC. We believe this achievement is because of the rank calculation technique of RAROF.

In heterogeneous low power and lossy networks, the routing technique is one of the main affecting factors for enhancing the performance of emergency monitoring IIoT application. The sensitivity of the 
application in terms of resource availability and reliability are extensively analyzed broadly in this paper. According to our study, we presume the proposed RAROF is very promising for mission-critical IoT applications due to its energy efficiency, stability, and reliability. Before our conclusion, we summarize the several relevant features of our proposition. The proposed multi-queuing traffic differentiation approach proved its effectiveness, and the approach of dealing with traffic sensitivity using the timer was reasonable. The strength and efficiency of IEMS rely on the RAROF provision of duty cycle adaptation and quantifying node's resource availability. The inter-packet interval, traffic generation rate, and transmission range of the node have a direct impact on the network performances, which can lead to more energy consumption, reliability degradation, and faster network partition. In addition, resource-ignoring path selection techniques can also affect the resource-constrained nodes, thereby disrupting overall network performance. In contrast to the default single metric based rank calculation and route selection technique, RAROF is based on multiple metrics including the duty cycle. Therefore, our resource aware route selection approach is more efficient and have significant influence on the network performance. Overall, with RAROF, the routing performance can be significantly improved for IoT based applications, and it is preferable than the existing OFs.

\section{Conclusions}

In this paper, to extend the lifetime of low power and lossy network and minimize the energy consumption, a new resource-aware and reliable objective function, RAROF, has been presented. In RAROF, both node characteristics and resource availability are taken into consideration to select the routing path. Reliable data communication is essential to ensure the ideal emergency monitoring system in different industries, for which the multi-queuing based traffic differentiation approach has been adopted that prioritizes the packets according to their sensitivity. With this approach, each node forwards the data packets to the channel of fixed-sized slots or reserved slots according to the traffic priority. In a heterogeneous network, the RAROF constructs routing path with the aim of extending node's life and minimizing energy consumption, paving the way towards green IIoT. Besides, a simple conceptual cloud-assisted IEMS architecture provides the promising potential functionality that collects, monitor, store, and analyze the data to reinforce the real-time emergency monitoring system. In this study, we found that the occurrence of network partition due to energy-constrained nodes increase when the nodes have low transmission range and inter-packet interval and high traffic flow. The simulation results obtained indicate that the proposed RAROF makes significant improvements in network lifetime, energy consumption, and reliability, as compared to OF-ER and OF-EC. During the study, we noticed that congestion is more likely to occur in resource-constrained networks due to fixed-sized channel slots. An efficient message scheduling technique is necessary to resolve this issue; however, it is part of our future work.

Author Contributions: K.S.B. conceptualized the proposed approach, performed an experimental investigation, and wrote the paper. G.H.C. supervised the work and review the manuscript. All authors have read and agreed to the published version of the manuscript.

Funding: This research received no external funding.

Acknowledgments: This work was supported by the research funds of Jeonbuk National University, in 2020.

Conflicts of Interest: The authors declare no conflict of interest.

\section{References}

1. Qin, W.; Chen, S.; Peng, M. Recent advances in Industrial Internet: Insights and challenges. Digit. Commun. Netw. 2020, 6, 1-13. [CrossRef]

2. Da Xu, L.; He, W.; Li, S. Internet of things in industries: A survey. IEEE Trans. Ind. Inform. 2014, 10, 2233-2243.

3. Liao, Y.; Loures, E.D.F.R.; Deschamps, F. Industrial Internet of Things: A systematic literature review and insights. IEEE Internet Things J. 2018, 5, 4515-4525. [CrossRef] 
4. $\quad$ Lucas-Estañ, M.C.; Sepulcre, M.; Raptis, T.P.; Passarella, A.; Conti, M. Emerging trends in hybrid wireless communication and data management for the industry 4.0. Electronics 2018, 7, 400. [CrossRef]

5. Wang, Q.; Jiang, J. Comparative examination on architecture and protocol of industrial wireless sensor network standards. IEEE Commun. Surv. Tutor. 2016, 18, 2197-2219. [CrossRef]

6. Zhang, W.; Liu, Y.; Han, G.; Feng, Y.; Zhao, Y. An energy efficient and QoS aware routing algorithm based on data classification for industrial wireless sensor networks. IEEE Access 2018, 6, 46495-46504. [CrossRef]

7. Han, G.; Zhou, L.; Wang, H.; Zhang, W.; Chan, S. A source location protection protocol based on dynamic routing in WSNs for the Social Internet of Things. Future Gener. Comput. Syst. 2018, 82, 689-697. [CrossRef]

8. Li, H.; Li, W.; Zhang, S.; Wang, H.; Pan, Y.; Wang, J. Page-sharing-based virtual machine packing with multi-resource constraints to reduce network traffic in migration for clouds. Future Gener. Comput. Syst. 2019, 96, 462-471. [CrossRef]

9. Wang, J.; Yang, Y.; Wang, T.; Sherratt, R.S.; Zhang, J. Big Data Service Architecture: A Survey. J. Internet Technol. 2020, 21, 393-405.

10. Zhang, J.; Zhong, S.; Wang, T.; Chao, H.C.; Wang, J. Blockchain-based systems and applications: A survey. J. Internet Technol. 2020, 21, 1-14.

11. Singh, S.; Ra, I.H.; Meng, W.; Kaur, M.; Cho, G.H. SH-BlockCC: A secure and efficient Internet of things smart home architecture based on cloud computing and blockchain technology. Int. J. Distrib. Sens. Netw. 2019, 15, 1550147719844159. [CrossRef]

12. Singh, P.; Nayyar, A.; Kaur, A.; Ghosh, U. Blockchain and Fog Based Architecture for Internet of Everything in Smart Cities. Future Internet 2020, 12, 61. [CrossRef]

13. Yaqoob, I.; Hashem, I.A.T.; Mehmood, Y.; Gani, A.; Mokhtar, S.; Guizani, S. Enabling Communication Technologies for Smart Cities. IEEE Commun. Mag. 2017, 55, 112-120. [CrossRef]

14. Kumar, V.; Tiwari, S. Routing in IPv6 over low-power wireless personal area networks (6LoWPAN): A survey. J. Comput. Netw. Commun. 2012, 2012. [CrossRef]

15. Kushalnagar, N.; Montenegro, G.; Schumacher, C.P. IPv6 over Low-Power Wireless Personal Area Networks (6LoWPANs): Overview, Assumptions, Problem Statement, and Goals. Available online: https://tools.ietf. org/html/rfc4919 (accessed on 16 May 2020).

16. Winter, T.; Thubert, P.; Brandt, A.; Hui, J.W.; Kelsey, R.; Levis, P.; Pister, K.; Struik, R.; Vasseur, J.P.; Alexander, R.K. RPL: IPv6 Routing Protocol for Low-Power and Lossy Networks. IETF RFC 6550. Available online: https://tools.ietf.org/html/rfc6550 (accessed on 16 May 2020).

17. Sobral, J.V.; Rodrigues, J.J.; Rabêlo, R.A.; Al-Muhtadi, J.; Korotaev, V. Routing protocols for low power and lossy networks in internet of things applications. Sensors 2019, 19, 2144. [CrossRef]

18. Tripathi, J.; De Oliveira, J.; Vasseur, J. Performance Evaluation of Routing Protocol for Low Power and Lossy Networks (RPL). IETF RFC 6687. Available online: https://tools.ietf.org/html/rfc6687 (accessed on 16 May 2020).

19. Raptis, T.P.; Passarella, A.; Conti, M. Performance analysis of latency-aware data management in industrial IoT networks. Sensors 2018, 18, 2611. [CrossRef]

20. Alnumay, W.S.; Chatterjee, P.; Ghosh, U. Energy aware secure routing for wireless ad hoc networks. IETE J. Res. 2014, 60, 50-59. [CrossRef]

21. Zhang, J.; Zhang, D.; Xie, K.; Qiao, H.; He, S. A VMIMO-based cooperative routing algorithm for maximizing network lifetime. China Commun. 2017, 14, 20-34. [CrossRef]

22. He, S.; Xie, K.; Xie, K.; Xu, C.; Wang, J. Interference-aware multisource transmission in multiradio and multichannel wireless network. IEEE Syst. J. 2019, 13, 2507-2518. [CrossRef]

23. Lim, C. A survey on congestion control for RPL-based wireless sensor networks. Sensors 2019, $19,2567$. [CrossRef]

24. Homaei, M.H.; Salwana, E.; Shamshirband, S. An enhanced distributed data aggregation method in the Internet of Things. Sensors 2019, 19, 3173. [CrossRef] [PubMed]

25. Almusaylim, Z.A.; Alhumam, A.; Mansoor, W.; Chatterjee, P.; Jhanjhi, N.Z. Detection and Mitigation of RPL Rank and Version Number Attacks in Smart Internet of Things. Preprints 2020, 2020070476. [CrossRef]

26. Kamgueu, P.O.; Nataf, E.; Ndie, T.D. Survey on RPL enhancements: A focus on topology, security and mobility. Comput. Commun. 2018, 120, 10-21. [CrossRef]

27. Lamaazi, H.; Benamar, N. A comprehensive survey on enhancements and limitations of the RPL protocol: A focus on the objective function. Ad Hoc Netw. 2020, 96, 102001. [CrossRef] 
28. Chen, D.; Varshney, P.K. QoS Support in Wireless Sensor Networks: A Survey. In Proceedings of the International Conference on Wireless Networks (ICWN), Las Vegas, NV, USA, 21-24 June 2004; pp. 1-7.

29. Hamid, Z.; Hussain, F.B. QoS in Wireless Multimedia Sensor Networks: A Layered and Cross-Layered Approach. Wirel. Pers. Commun. 2014, 75, 729-757. [CrossRef]

30. Yigitel, M.A.; Incel, O.D.; Ersoy, C. QoS-Aware MAC Protocols for Wireless Sensor Networks. Comput. Netw. 2011, 55, 1982-2004. [CrossRef]

31. Hasan, M.Z.; AI-Rizzo, H.; AI-Turjman, F. A Survey on Multipath Routing Protocols for QoS Assurances in Real-time Wireless Multimedia Sensor Networks. IEEE Commun. Surv. Tutor. 2017, 19, 1424-1456. [CrossRef]

32. AI-Turjman, F. Cognitive Routing Protocol for Disaster-inspired Internet of Things. Future Gener. Comput. Syst. 2019, 92, 1103-1115. [CrossRef]

33. Tang, W.; Ma, X.; Huang, J.; Wei, J. Toward Improved RPL: A Congestion Avoidance Multipath Routing Protocol with Time Factor for Wireless Sensor Networks. J. Sens. 2016, 2016, 1-11. [CrossRef]

34. Zhu, C.; Leung, V.C.; Shu, L.; Ngai, E.C.H. Green internet of things for smart world. IEEE Access 2015, 3, 2151-2162. [CrossRef]

35. Arshad, R.; Zahoor, S.; Shah, M.A.; Wahid, A.; Yu, H. Green IoT: An investigation on energy saving practices for 2020 and beyond. IEEE Access 2017, 5, 15667-15681. [CrossRef]

36. Huang, J.; Meng, Y.; Gong, X.; Liu, Y.; Duan, Q. A novel deployment scheme for green internet of things. IEEE Int. Things J. 2014, 1, 196-205. [CrossRef]

37. Misra, S.; Chatterjee, S.; Obaidat, M.S. On theoretical modeling of sensor cloud: A paradigm shift from wireless sensor network. IEEE Syst. J. 2014, 11, 1084-1093. [CrossRef]

38. Capone, S.; Brama, R.; Accettura, N.; Striccoli, D.; Boggia, G. An Energy Efficient and Reliable Composite Metric for RPL Organized Networks. In Proceedings of the International Conference on Embedded and Ubiquitous Computing, Milano, Italy, 26-28 August 2014; pp. 178-184.

39. Alishahi, M.; Moghaddam, M.H.Y.; Pourreza, H.R. Multi-class Routing Protocol Using Virtualization and SDN-enabled Architecture for Smart Grid. Peer Peer Netw. Appl. 2018, 11, 380-396. [CrossRef]

40. Murali, S.; Jamalipour, A. Mobility-aware energy-efficient parent selection algorithm for low power and lossy networks. IEEE Internet Things J. 2018, 6, 2593-2601. [CrossRef]

41. Ullah, R.; Faheem, Y.; Kim, B.S. Energy and congestion-aware routing metric for smart grid AMI networks in smart city. IEEE Access 2017, 5, 13799-13810. [CrossRef]

42. Bhandari, K.S.; Cho, G.H. A Resource Oriented Route Selection Framework Using Contextual Information Based on Fuzzy Logic. Electronics 2019, 8, 1023. [CrossRef]

43. Alvi, S.A.; Shah, G.A.; Mahmood, W. Energy efficient green routing protocol for internet of multimedia things. In Proceedings of the International Conference on Intelligent Sensors, Sensor Networks and Information Processing (ISSNIP), Singapore, 7-9 April 2015; pp. 1-6.

44. Lamaazi, H.; Benamar, N. OF-EC: A novel energy consumption aware objective function for RPL based on fuzzy logic. J. Netw. Comput. Appl. 2018, 117, 42-58. [CrossRef]

45. Singh, P.; Chen, Y.C. RPL Enhancement for a Parent Selection Mechanism and an Efficient Objective Function. IEEE Sens. J. 2019, 19, 10054-10066. [CrossRef]

46. Toscano, E.; Bello, L.L. Multichannel superframe scheduling for IEEE 802.15. 4 industrial wireless sensor networks. IEEE Trans. Inf. 2011, 8, 337-350. [CrossRef]

47. Pavkovic, B.; Hwang, W.J.; Theoleyre, F. Cluster-directed acyclic graph formation for IEEE 802.15. 4 in multihop topologies. In Proceedings of the International Conference on New Technologies, Mobility and Security (NTMS), Istanbul, Turkey, 7-10 May 2012; pp. 1-6.

48. Yang, Y.; Wang, J. Design guidelines for routing metrics in multihop wireless networks. In Proceedings of the International Conference on Computer Communication (INFOCOM), Phoenix, AZ, USA, 13-18 April 2008; pp. 1615-1623.

49. Cao, Y.; Wu, M. A novel RPL algorithm based on chaotic genetic algorithm. Sensors 2018, 18, 3647. [CrossRef]

50. Hassan, A.; Alshomrani, S.; Altalhi, A.; Ahsan, S. Improved routing metrics for energy constrained interconnected devices in low-power and lossy networks. J. Commun. Netw. 2016, 18, 327-332.

51. Hassan, M.N.; Murphy, L.; Stewart, R. Traffic differentiation and dynamic duty cycle adaptation in IEEE 802.15. 4 beacon enabled WSN for real-time applications. Telecommun. Sys. 2016, 62, 303-317. [CrossRef]

52. IEEE 802.15.4 Compliant CC2420 Data Sheet [online]. Available online: https://www.ti.com/product/CC2420 (accessed on 19 August 2020). 
53. Djenouri, D.; Balasingham, I. Traffic-differentiation-based modular QoS localized routing for wireless sensor networks. IEEE Trans. Mob. Comput. 2010, 10, 797-809. [CrossRef]

54. Alazzawi, L.; Elkateeb, A. Performance evaluation of the WSN routing protocols scalability. J. Comput. Syst. Netw. Commun. 2008, 2008. [CrossRef]

(C) 2020 by the authors. Licensee MDPI, Basel, Switzerland. This article is an open access article distributed under the terms and conditions of the Creative Commons Attribution (CC BY) license (http://creativecommons.org/licenses/by/4.0/). 